\title{
Large-scale phylogenomics of the genus Macrostomum (Platyhelminthes) reveals cryptic diversity and novel sexual traits
}

\author{
Journal Article \\ Author(s): \\ Brand, Jeremias N.; Viktorin, Gudrun; Wiberg, R. Axel W.; Beisel, Christian; Schärer, Lukas \\ Publication date: \\ 2022-01 \\ Permanent link: \\ https://doi.org/10.3929/ethz-b-000517322
}

Rights / license:

Creative Commons Attribution 4.0 International

Originally published in:

Molecular Phylogenetics and Evolution 166, https://doi.org/10.1016/j.ympev.2021.107296 


\title{
Large-scale phylogenomics of the genus Macrostomum (Platyhelminthes) reveals cryptic diversity and novel sexual traits
}

\author{
Jeremias N. Brand $^{\mathrm{a},{ }^{*}, 1}$, Gudrun Viktorin ${ }^{\mathrm{a}}$, R. Axel W. Wiberg ${ }^{\mathrm{a}}$, Christian Beisel ${ }^{\mathrm{b}}$, \\ Lukas Schärer $^{\mathrm{a}}$ \\ ${ }^{\text {a }}$ University of Basel, Department of Environmental Sciences, Zoological Institute, Vesalgasse 1, 4051 Basel, Switzerland \\ ${ }^{\mathrm{b}}$ Department of Biosystems Science and Engineering, ETH Zürich, Basel, Switzerland
}

\section{A R T I C L E I N F O}

\section{Keywords:}

Phylogenetics

Evolution

Cryptic speciation

Transcriptome

Taxonomy

Platyhelminths

\begin{abstract}
A B S T R A C T
Free-living flatworms of the genus Macrostomum are small and transparent animals, representing attractive study organisms for a broad range of topics in evolutionary, developmental, and molecular biology. The genus includes the model organism $M$. lignano for which extensive molecular resources are available, and recently there is a growing interest in extending work to additional species in the genus. These endeavours are currently hindered because, even though $>200$ Macrostomum species have been taxonomically described, molecular phylogenetic information and geographic sampling remain limited. We report on a global sampling campaign aimed at increasing taxon sampling and geographic representation of the genus. Specifically, we use extensive transcriptome and single-locus data to generate phylogenomic hypotheses including 145 species. Across different phylogenetic methods and alignments used, we identify several consistent clades, while their exact grouping is less clear, possibly due to a radiation early in Macrostomum evolution. Moreover, we uncover a large undescribed diversity, with 94 of the studied species likely being new to science, and we identify multiple novel morphological traits. Furthermore, we identify cryptic speciation in a taxonomically challenging assemblage of species, suggesting that the use of molecular markers is a prerequisite for future work, and we describe the distribution of putative synapomorphies and suggest taxonomic revisions based on our finding. Our large-scale phylogenomic dataset now provides a robust foundation for comparative analyses of morphological, behavioural and molecular evolution in this genus.
\end{abstract}

\section{Introduction}

The genus Macrostomum (Macrostomorpha, Platyhelminthes) is a large clade of free-living flatworms with a global distribution in marine, brackish and freshwater environments (Ferguson, 1954). After the Catenulida, the Macrostomorpha are the earliest-branching lineage of Platyhelminthes (Laumer et al., 2015), giving Macrostomum an interesting taxonomic position, and representing a valuable point of comparison to other well-studied flatworms such as members of the Tricladida and the parasitic Neodermata. The genus contains the model organism M. lignano (Ladurner et al., 2005; Wudarski et al., 2020), which, in part due to its high transparency and experimental amenability, is used for the study of a variety of biological topics, including aging (Mouton et al., 2018), bioadhesion (Lengerer et al., 2016; Wunderer et al., 2019), regeneration and stem cells (Egger et al., 2006;
Grudniewska et al., 2016; Lengerer et al., 2016), and sexual selection (Schärer et al., 2011; Marie-Orleach et al., 2021). A rich experimental and genomic toolset is available for M. lignano, including in situ hybridization, RNA interference, transgenesis, a range of transcriptomes, and a sequenced genome (Pfister et al., 2007, 2008; Wasik et al., 2015; Wudarski et al., 2017). Perhaps as a result of the emergence of Macrostomum as a promising model organism, there has been a resurgence of taxonomic research on this genus and related taxa (e.g. Ladurner et al., 2005; Adami et al., 2012; Janssen et al., 2015; Sun et al., 2015; Atherton et al., 2019; Xin et al., 2019; Schärer et al., 2020), after an initially very active period of research in the middle of the last century (e.g. Luther, 1947, 1960; Ferguson, 1954).

Moreover, primarily due to the discovery of a whole genome duplication and accompanying karyotype instability in M. lignano (Zadesenets et al., 2016, 2017), there is increased interest in expanding the

\footnotetext{
* Corresponding author at: University of Basel, Department of Environmental Sciences, Zoological Institute, Vesalgasse 1, 4051 Basel, Switzerland.

E-mail address: jeremias.br@gmail.com (J.N. Brand).

1 Current address: Max Planck Institute for Biophysical Chemistry Department of Tissue Dynamics and Regeneration, Am Fassberg 11, 37077 Göttingen, Germany.
} 
genomic resources and molecular phylogenetic placement of additional species in the genus,. To this end, recent work has provided transcriptomic resources for additional species (Brand et al., 2020) and fieldcollections have revealed similar karyological rearrangements to the ones observed in $M$. lignano in some closely related species, while others were found to have stable diploid genomes (Schärer et al., 2020; Zadesenets et al., 2020). Accurate phylogenetic information will greatly help guide the search for additional Macrostomum model species. And a better phylogeny will also permit to study the (co)evolution of the large diversity of morphological and behavioural traits in this genus (see below).

Members of the flatworm genus Macrostomum have intriguing aflagellate sperm that in many species carry two stiff lateral bristles and a terminal brush (Fig. 1A), while other species have sperm that lack these structures (Fig. 1B). Moreover, species show a large diversity in both the morphology of the stylet (Fig. 2) and the female antrum. Previous work has also shown that there are two main clades of Macrostomum that differ in terms of their mating behaviour (Schärer et al., 2011). One clade (called "Clade I" in Schärer et al., 2011) was characterized by a stereotypical stylet and sperm morphology, and species in that clade are thought to mate exclusively through hypodermic insemination, a form of traumatic mating where the ejaculate is injected though the mating partner's epidermis into the tissue using a needle-like copulatory organ (Fig. 1B). The second clade (called "Clade II" in Schärer et al., 2011) contains species that are thought to mate primarily through reciprocal copulation, where both individuals reciprocally insert their (often blunt) stylet (Fig. 1A) into the female antrum of the mating partner, and are able to donate and receive ejaculate at the same time (see also Schärer et al., 2004, 2011; Singh et al., 2020). Within Clade II one species, $M$. hystrix, was found to have convergently evolved the hypodermic mating behaviour, and this was associated with convergent changes in stylet, sperm, and antrum morphology, leading to a morphology that is very similar to that of M. pusillum (Fig. 1B). Understanding the molecular phylogeny of the genus will furthermore shed light on the validity of several taxonomic groups that have been erected among the Macrostomidae solely based on morphological synapomorphies (e.g. An-derLan, 1939; Ferguson, 1954; Faubel et al., 1994). Specifically, recent work suggests that there is widespread convergent evolution of numerous reproductive morphology traits, including the stylet (male intromittent organ), the sperm, the female antrum (female sperm receiving organ) (Schärer et al., 2011), as well as the presence of a second female opening (Schärer et al., 2011; Xin et al., 2019). If we were to find that traits previously considered to be useful taxonomic characters have actually originated frequently, thus possibly rendering several of the putative synapomorphies homoplastic, this would indicate that these taxonomic groups need to be revaluated.

In the current study, we present results from a global sampling effort to increase both taxon sampling and geographic representation of the genus. We also present a phylogenomic analysis based on a combination of transcriptome and Sanger-sequencing, thereby significantly expanding the phylogenetic and genomic resources available for the genus.

\section{(A) Macrostomum cliftonense}

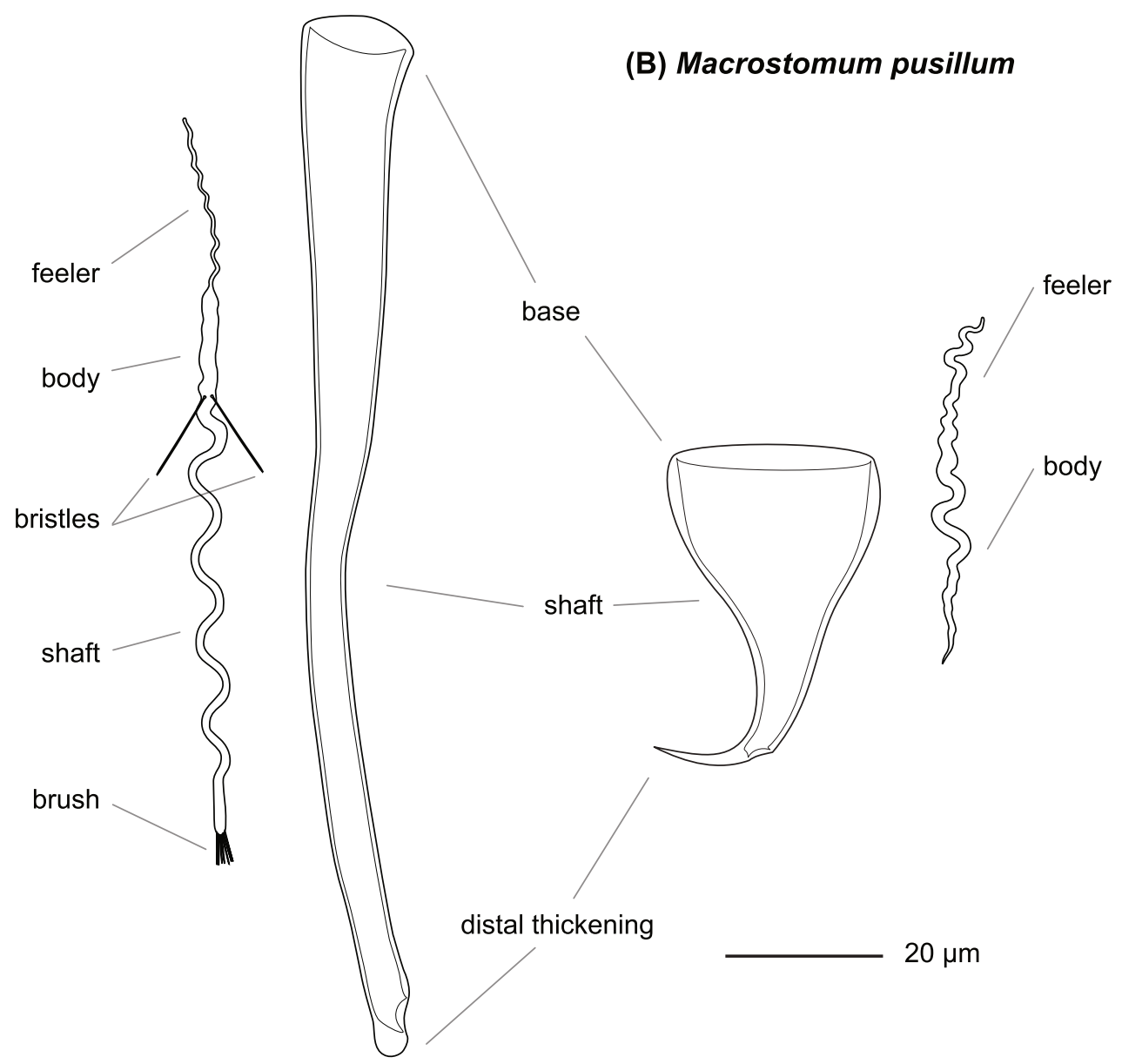

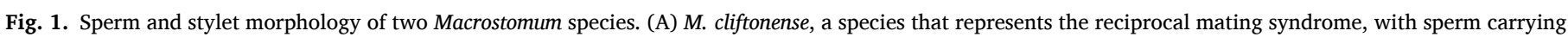

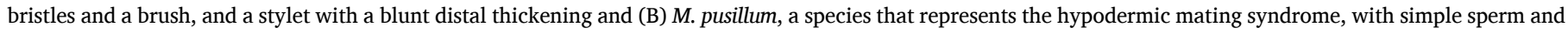
a needle-like stylet. 


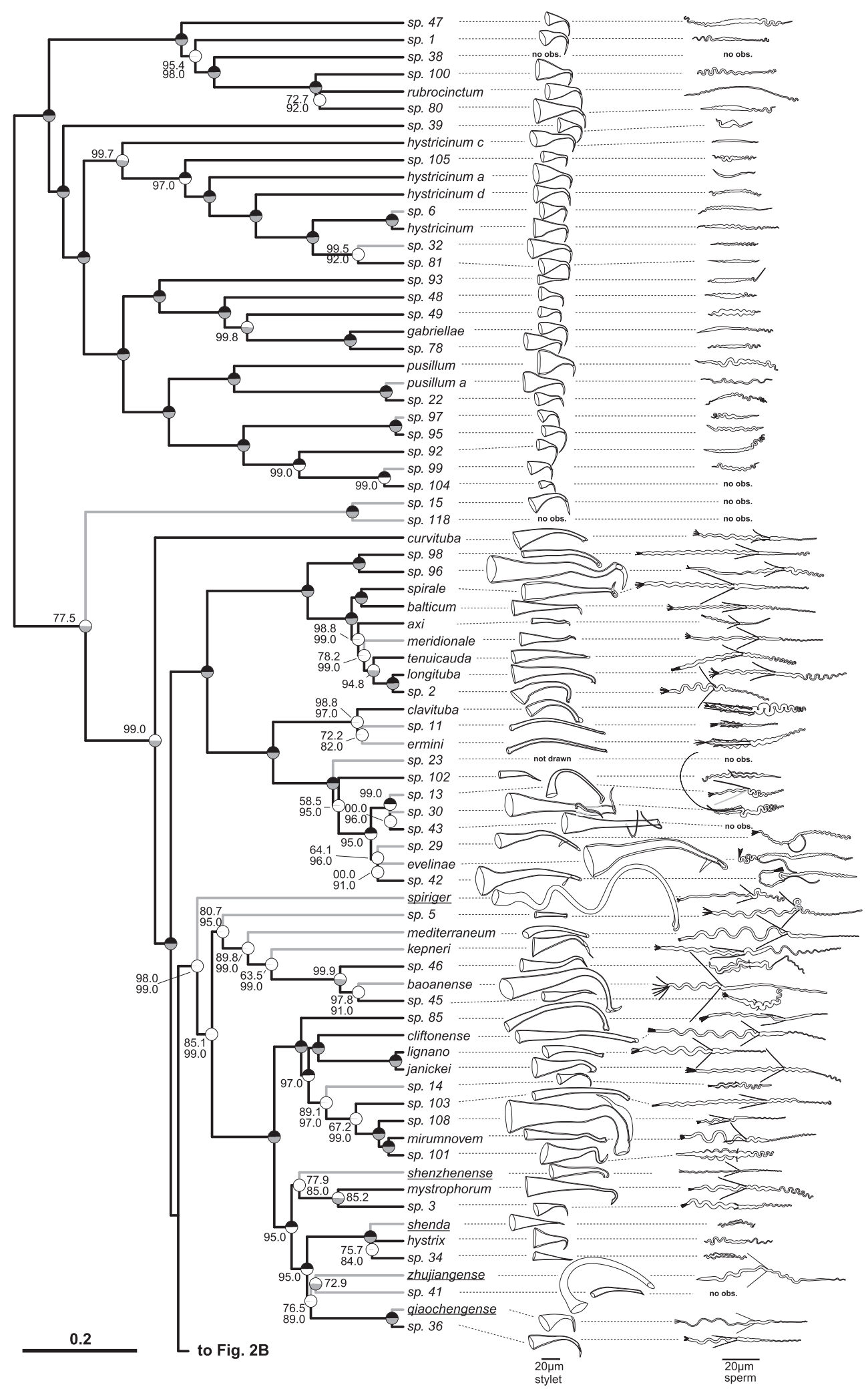

Fig. 2. Phylogeny of the genus Macrostomum, showing the striking diversity in stylet and sperm morphology across the genus. The ultrametric phylogeny (C-IQTREE) includes all 145 studied species (with 77 species depicted in Fig. 2A and 68 species in Fig. 2B), while additional phylogenies based solely on transcriptomes are shown in Fig. 3 and Fig. S2. Branch supports are ultrafast bootstraps (top, black if 100) and approximate likelihood ratio tests (bottom, grey if 100). Species without available transcriptomes that were included in C-IQ-TREE based on a $28 S$ rRNA fragment are indicated with grey branches. Stylet and sperm are drawn based on our live observations, except for species with underlined names, which were redrawn based on the species descriptions (M. acus, M. obtusa and M. sinense from Wang 2005; M. heyuanense and M. bicaudatum from Sun et al., 2015; M. chongqingense and M. zhaoqingense from Lin et al., 2017a; M. shiyanense and M. lankouense from Lin et al., 2017b; M. shenzhenense and M. qiaochengense from Wang et al., 2017; and M. spiriger and M. shenda from Xin et al., 2019). Note that the stylet of M. sp. 15 is not drawn to scale, the stylets of some species are drawn at half size (stylet 1/2), and the stylet of M. sp. 23 is not drawn since it was incomplete (specimen ID MTP LS 913). Unobserved structures are marked as no observation (no obs.). 


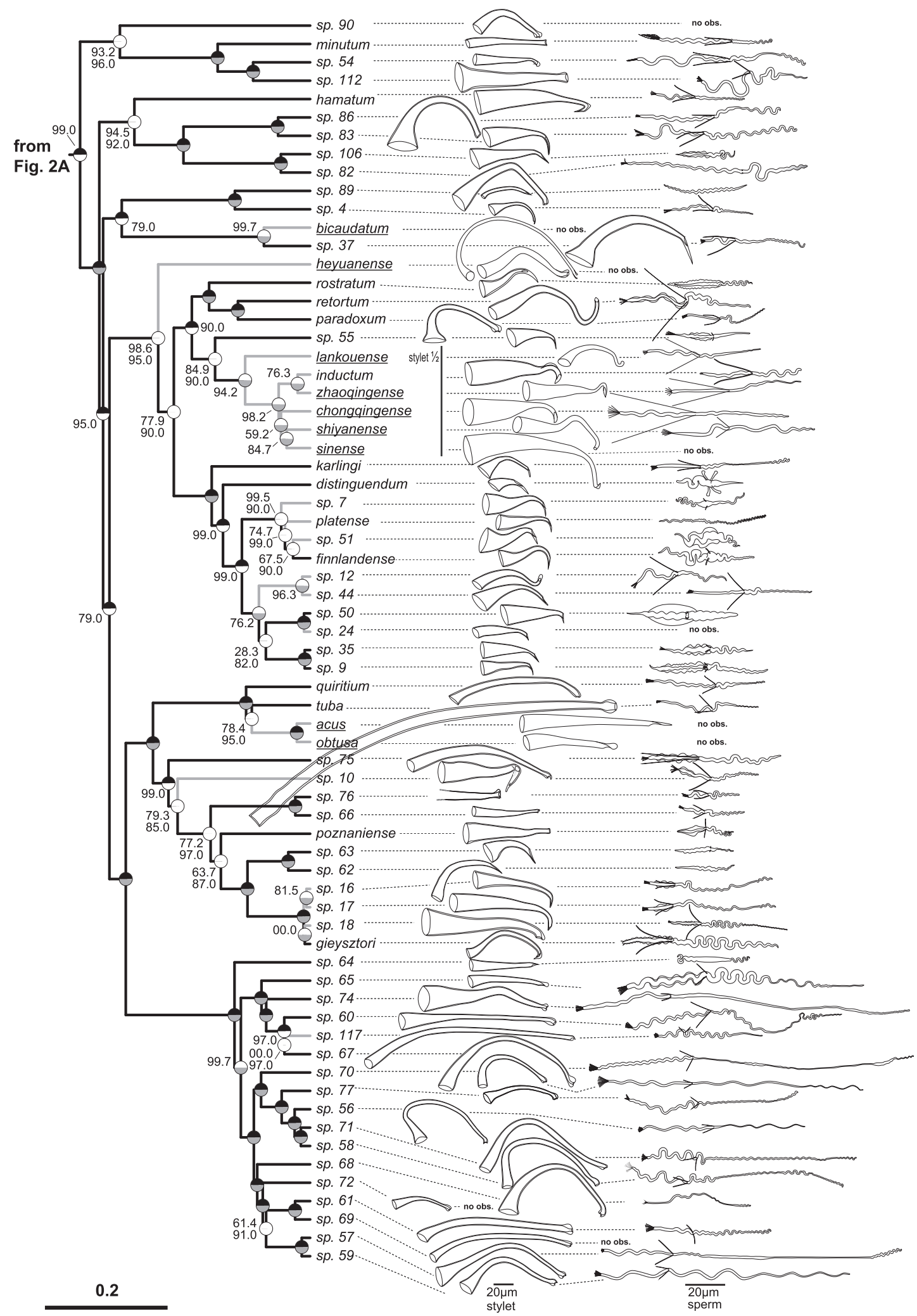

Fig. 2. (continued).

\section{Material and methods}

\subsection{Included species and documentation}

In a global sampling campaign, we collected $>1600$ specimens of 131 Macrostomum species (see species delimitation section below) and additionally included data on 14 species from the literature. Almost all specimens were collected from the field in freshwater, brackish or marine habitats, either from sediment samples or from water plants (for details on specimens see Table S1). We documented specimens extensively with digital photomicrography, as previously described (Ladurner et al., 2005; Schärer et al., 2011; Janssen et al., 2015), using light 
microscopes (Leica DM2500, Olympus BH2, Leitz Diaplan, Zeiss Axioscope 5) with differential interference contrast (DIC) and equipped with digital cameras (Sony DFW-X700, Sony DFK41 and Ximea xiQ MQ042CG-CM). We collected both images and videos at various magnifications (40x to 1000x), documenting the general habitus and details of internal organs, which is possible due to the small size and high transparency of these organisms. To document sperm morphology, we amputated a worm's tail slightly anterior of the seminal vesicle, ruptured the seminal vesicle (as described in Janicke and Schärer, 2010), and documented the released sperm in a smash preparation using DIC and sometimes also phase-contrast microscopy. When possible, we prepared whole-mount permanent preparations of these amputated tails to preserve a physical specimen of the male intromittent organ, the stylet (Schärer et al., 2011; Janssen et al., 2015). Finally, we preserved the entire animal, or its anterior portion when amputated, for molecular analysis, in either RNAlater solution (Sigma, stored at $4{ }^{\circ} \mathrm{C}$ up to a few weeks and then at $-80^{\circ} \mathrm{C}$ ) or in absolute ethanol (stored cool for up to a few weeks and then at $-20^{\circ} \mathrm{C}$ ).

\subsection{Sequence data generation}

We extracted both DNA and RNA from the RNAlater samples using the Nucleospin XS Kit in combination with the Nucleospin RNA/DNA Buffer Set (Macherey-Nagel), and we extracted DNA from the ethanol samples using the DNeasy Blood and Tissue kit (Qiagen, Germany). Extracted DNA and RNA was stored at $-80{ }^{\circ} \mathrm{C}$. We amplified a partial $28 S$ rRNA sequence from DNA samples using PCR primers ZX-1 and 1500R and, for some fragments, additional nested PCR using primers ZX-1 and 1200R, or 300F and 1500R, with polymerases and cycling conditions as previously described (Schärer et al., 2011, 2020). For some fragments, we obtained additional sequences using internal primers 1090F and ECD2 (both Schärer et al., 2011). We sequenced the resulting fragments from both sides using the PCR primers (Microsynth, Switzerland), assembled them in Geneious (v 11.1.5) with the built-in assembler (using the Highest sensitivity setting), with minor manual trimming and correction. The final assembled sequences had a length between 942 and 1172 bp (see GenBank accessions MT428556MT429159).

We selected 145 RNA samples for RNA-Seq library generation. For 144 RNA samples, we generated RNA-Seq libraries from single specimens using SMART-Seq v4 (Clontech Laboratories, Inc.) combined with the Nextera XT DNA library preparation kit (Illumina). When at least 5 ng total RNA was available, we performed the SMART-Seq v4 protocol with 12 amplification cycles and otherwise used $1 \mathrm{ng}$ and performed 15 amplification cycles. To generate the RNA-Seq library for $M$. clavituba we collected 40 specimens from one sample location and for each specimen confirmed the species assignment through observation of the species-specific stylet morphology using DIC microscopy. We then extracted RNA from these 40 pooled animals using $\mathrm{Tri}^{\mathrm{TM}}$ reagent (Sigma) and prepared the library using the TruSeq ${ }^{\circledR}$ Stranded mRNA kit (Illumina). All amplified cDNA and final library constructs were checked for quality using a Fragment Analyzer (Agilent) and then sequenced as 101 paired-end reads on the HiSeq2500 platform (using the HiSeq ${ }^{\circledR}$ SBS Kit v4, Illumina) at the Genomics Facility Basel of the University of Basel and the Department of Biosystems Science and Engineering of the ETH Zürich.

\subsection{Species delimitation}

Most collected specimens could not be assigned to a taxonomically described Macrostomum species (http://turbellaria.umaine.edu, Tyler et al., 2006) and are likely new to science. We present transcriptomelevel information for most species, but it was not feasible to conduct RNA-Seq on all $>1600$ collected specimens. To facilitate specimen assignment, as well as comparative analysis of morphological traits across the genus that we will report on in a separate article (Brand et al.,
2021), we, therefore, implemented a species delimitation approach that was independent of the RNA-Seq data. Specifically, we relied on morphology and the partial $28 S$ rRNA sequences for species assignment. This $28 S$ rRNA fragment is widely used as a DNA barcode for flatworms (e.g. de Chambrier et al., 2004, 2015; Janssen et al., 2015; Scarpa et al., 2015). We used 668 sequences, of which 604 were generated for this study (Accessions: MT428556-MT429159) and 64 were taken from public databases (see also Table S1), representing the available diversity across the genus. We also included 16 sequences from seven species of our chosen outgroup genus Psammomacrostomum (see Table S1). We aligned sequences using MAFFT ("-genafpair -maxiterate 1000") and removed all columns that contained at least one gap leaving us with an alignment with 787 sites and 385 variable bases. Next, we generated haplotype networks for the recovered clades using the TCS algorithm (Templeton et al., 1992) implemented in the TCS software (Clement et al., 2000). We thus ignore indels to avoid the generation of cryptic species solely based on these, since scoring indels is non-trivial, and they are usually treated as missing data (Simmons et al., 2007). We chose to delimit species with at least 4 mutational differences in the network. Such a difference in this $28 S$ rRNA fragment is sometimes considered as indicative of distinct species among microturbellarians (Scarpa et al., 2015), but in our own experience recently diverged species within Macrostomum may show less divergence (Schärer et al., 2020). Additional markers like mitochondrial COI are frequently used to detect recent divergence (e.g. Schärer et al., 2011; Janssen et al., 2015). But despite considerable efforts, including trying to take advantage of mitochondrial sequences from the available transcriptome assemblies, we were unable to develop universal COI primers for the entire genus Macrostomum (a common issue in flatworms, see e.g. Vanhove et al., 2013) and individual primer optimization, as in Schärer et al. (2020), was not feasible here for each species. We, therefore, chose to additionally delimit species with 3 or fewer differences in this $28 S$ rRNA fragment, provided that they showed clear diagnostic differences in morphology. Specifically, when a species' morphology was diagnostic, we sequenced several specimens, if possible from different sample locations, to confirm that they were indeed molecularly similar and then assigned additional specimens based on morphology only. When no specific diagnostic traits could be defined (as was the case for many species of the hypodermic clade), we sequenced all specimens collected for molecular assignment. We provide haplotype networks accompanied by drawings of the diagnostic features for all species in the supporting information (SI Species delimitation).

\subsection{Transcriptome selection}

We used 134 transcriptomes representing 105 species, including four distant outgroups, Haplopharynx rostratum, Microstomum lineare, Myozonaria bistylifera, and Karlingia sp. 1, three species from the sister genus, Psammomacrostomum (see also Janssen et al., 2015), and 98 Macrostomum species in the phylogenomic analysis. This included the four publicly available high-quality transcriptomes of M. hystrix, M. spirale, M. pusillum (Brand et al., 2020), and M. lignano (Wudarski et al., 2017; Grudniewska et al., 2018), as well as 130 de novo assembled transcriptomes. Transcriptomes were assembled as previously described (Brand et al., 2020) and were mostly derived from whole single specimens and a few from anterior fragments only, which may reduce the transcriptome repertoire. Eight transcriptomes were assembled from combined single worm RNA-Seq data sets collected at the same location and assigned to the species based on our taxonomic expertise and the detailed image and video material we deposit for all specimens. Finally, the transcriptome of $M$. clavituba was assembled from RNA extracted from 40 pooled animals collected from the same location (for details see Table S2).

We determined the empirical insert size of our libraries by mapping the reads to the assemblies using SNAP (version 1.0, Zaharia et al., 2011) and then extracting the mean insert size using Picard (version 2.20.2). 
We assessed transcriptome quality using TransRate (version 1.0.2, Smith-Unna et al., 2016), which maps the reads back to the assembly and calculates mapping metrics, and BUSCO (version 2.0, Waterhouse et al., 2017, 978 genes in the metazoan dataset, version 2016-11-01), which searches for the presence of a curated set of core conserved genes. BUSCO scores were also used to select one representative transcriptome when multiple transcriptomes were available for a species (see below).

\subsection{Orthology inference}

To infer a set of orthologous genes (orthologs) we predicted open reading frames (ORFs) for each transcriptome using TransDecoder (version 5.3.0, Haas et al., 2013) with Pfam searches (version 32.0) to retain transcripts with predicted proteins and kept only one ORF per transcript using the "single_best_only" option. We then clustered predicted proteins with at least $99.5 \%$ sequence identity using the CD-HIT clustering algorithm (version 4.7, Fu et al., 2012). The amino acid sequences were then processed with OrthoFinder (version 2.2.6, Emms and Kelly, 2015) using the "-os" option to perform length-adjusted reciprocal BLAST searches, followed by MCL clustering. We processed the resulting set of homologous genes (homogroups) using modified scripts from the phylogenomic dataset construction workflow (Yang and Smith, 2014). We aligned all homogroups that contained at least 10 species using MAFFT (version 7.310, “-genafpair -maxiterate 1000", Nakamura et al., 2018), inferred the best substitution model with ModelFinder (Kalyaanamoorthy et al., 2017), and the gene tree using IQ-TREE (version 1.5.5, Nguyen et al., 2015, command: "-mset DCMut, JTTDCMut, LG, mtZOA, PMB, VT, WAG -mfreq FU,F -mrate G“). Then we trimmed the gene trees using "trim_tips.py" to remove tip branches that were longer than two substitutions per site and "mask_tips_by_taxonID_transcripts.py" to remove monophyletic paralogs by choosing the sequence with the best representation in the alignment. We split off subtrees with long internal branches using "cut_long_internal_branches. py" and inferred orthologs using the rooted outgroup method in "prune_paralogs_RT.py“. This method uses known outgroup taxa to root the phylogenies, which then allows to infer the history of speciation and duplication and extract the most inclusive set of orthologs. We defined Haplopharynx rostratum, Microstomum lineare, Myozonaria bistylifera, and Karlingia sp. 1 as the outgroup and all Macrostomum and Psammomacrostomum as an ingroup, since the algorithm does not include the defined outgroup in the output, and we then used Psammomacrostomum to root our final ortholog trees. Next, we realigned the orthogroups using MAFFT and trimmed the alignment with ZORRO (Wu et al., 2012), discarding any columns in the alignment with a score of less than five and filtering alignments that were shorter than 50 amino acids after trimming. Finally, we inferred ortholog gene trees with 100 nonparametric bootstraps with IQ-TREE, by inferring the best fitting model ("-mset DCMut, JTTDCMut, LG, mtZOA, PMB, VT, WAG -mfreq FU,F -mrate E,I,G,I + G“). These best fitting substitution models were later also used for the partitioned maximum-likelihood analysis. We generated two gene matrices, one matrix containing many genes but a relatively moderate occupancy (called L for low occupancy) and one with a lower gene number but a higher occupancy (called $\mathrm{H}$ for high occupancy, Table 1).

\subsection{Phylogenetics}

We conducted most analyses on the $\mathrm{H}$ alignment for computational tractability and since missing data can have a negative influence on tree inference, particularly when using Bayesian methods (Roure et al., 2013, but see Tan et al., 2015), and only the less computationally demanding summary and maximum-likelihood methods were also applied to the L alignment. So for both alignments, we calculated a maximum likelihood species phylogeny using IQ-TREE ("L-IQ-TREE" and "H-IQ-TREE") with a partition for each gene and using the best
Table 1

Characteristics of the protein alignments used for phylogenetic inference. We used one alignment aimed at a high number of genes (L alignment) and one alignment aimed at high occupancy ( $\mathrm{H}$ alignment). The alignments used were trimmed to only include regions with a high probability of being homologous using ZORRO, and the statistics are given for the alignments before and after trimming.

\begin{tabular}{lllll}
\hline Alignment metrics & $\begin{array}{l}\text { L } \\
\text { untrimmed }\end{array}$ & L & $\begin{array}{l}\text { H } \\
\text { untrimmed }\end{array}$ & $\mathrm{H}$ \\
\hline No. genes & 8128 & 8128 & 385 & 385 \\
No. amino acids AA & $5,057,157$ & $1,687,014$ & 200,729 & 94,625 \\
$\begin{array}{l}\text { No. variable sites } \\
\text { No. parsimony }\end{array}$ & $3,263,955$ & $1,157,689$ & 135,887 & 74,175 \\
$\quad$ informative sites & $2,287,246$ & 934,803 & 103,425 & 63,066 \\
Missing data (\%) & 78.1 & 59.3 & 55.7 & 22.9 \\
\hline
\end{tabular}

substitution model for each gene (see above). We calculated 1000 ultrafast bootstraps and conducted an approximate-likelihood ratio test to assess branch support.

Additionally, we inferred Bayesian phylogenies using ExaBayes (version 1.5, Aberer et al., 2014) for the H alignment ("H-ExaBayes") with partitions for each gene, equal prior probability on all available amino acid substitution models, and with gamma models for all partitions. We ran four independent chains retaining every 500th iteration and discarding the first 365,000 iterations as burn-in. We terminated the analysis after 1.46 million generations since the average deviation of split frequencies between all the chains was below $1 \%$, indicating convergence. However, an assessment of convergence using Gelman's R of the likelihoods with the R package coda (Plummer et al., 2006), suggested that only three chains had converged, while one appeared stuck on a lower likelihood peak. Since all chains quickly converged on the same topology, the local peak probably occurs due to differences in the substitution models applied, with the HIVB model being present at a low rate in the three converged chains, but being absent in the fourth. To guard against model misspecification, we discarded the fourth chain and combined the three converged chains to calculate a consensus tree using quadratic path distance optimization (using the ls.consensus function in the $\mathrm{R}$ package phytools).

To construct phylogenies while accounting for potential incomplete lineage sorting, we ran the quartet based method implemented in ASTRAL-III (version 5.6.1, Zhang et al., 2018) on both alignments ("LASTRAL" and "H-ASTRAL"). We assessed the level of gene tree-species tree conflict at each node of the phylogeny using the quartet support score, which gives the proportion of quartet trees induced by the gene trees that are supporting the species tree topology as opposed to the two possible alternatives (Zhang et al., 2018). Strong support for the species tree partition can be interpreted as little disagreement between the gene trees, while support for the alternate topologies indicates strong gene tree - species tree conflict. Furthermore, we evaluated discordance between our species tree phylogenies using the Robinson-Foulds distance, calculated as implemented in the phangorn $\mathrm{R}$ package (version 2.5.5).

We also chose representative 28S rRNA sequences from the haplotype network analysis to infer phylogenetic placement for species without a transcriptome. For species with a transcriptome, we mostly chose the 28S rRNA sequence derived from the same specimen (for exceptions see Table S2). We aligned the sequences using MAFFT ("-maxiterate 1000 -globalpair"), trimmed the start and end using trimAl ("-nogaps -terminalonly") (Capella-Gutierrez et al., 2009) and determined the best substitution model using ModelFinder with the BIC criterion. We then combined this alignment with the $\mathrm{H}$ alignment, and analysed it with the best fitting substitution model using IQ-TREE as described above (referred to as "C-IQ-TREE", called C for combined). To facilitate comparative analysis, we transformed the phylogenies (C-IQTREE, H-IQ-TREE and H-ExaBayes) to be ultrametric and with a root depth of 1 using the penalized marginal likelihood approach (Sanderson, 2002) implemented in the software TreePL (Smith and O'Meara, 2012). 
After all of the above analyses were completed, we discovered that some transcripts of the RNA-Seq libraries constructed with the SMARTSeq v4 cDNA kit contained cDNA synthesis primer sequences. We did not remove these primers before transcriptome assembly since (i) the manufacturer specifically states they should not occur in RNA-Seq reads if used in combination with the Nextera XT DNA library preparation kit (Appendix C in SMART-Seq v4 Ultra Low Input RNA Kit for Sequencing User Manual, Takara Bio Inc. available at: https://www.takarabio. com/assets/a/114825) and (ii) because the relevant primer sequence is proprietary (both confirmed by Takara EU tech support in November 2020), so that we initially did not have access to its sequence. We only discovered the offending primer sequence due to our work on two ongoing Macrostomum genome projects. Because the analyses presented here had at that point used approximately 650,000 CPU hours of computation, and since we expected the effect of that primer to be small, we elected to perform follow-up analyses to determine how robust our results were to its removal, rather than opting for a complete reassembly and re-analysis of all the data. As we outline in the supporting information (SI Primer removal), the removal of the cDNA synthesis primer sequences had little effect on the topology and branch lengths of L-IQ-TREE, H-IQ-TREE and C-IQ-TREE. Since our results appear to be robust, we here focus on the results from our initial analysis.

\subsection{Ancestral state reconstruction}

We reconstructed the ancestral state of the salinity of the habitat from which our specimens were collected. During our field collections we generally measured the salinity of the samples in \%o (permille) using a hand refractometer (HRS 16, Krüss Optronic GmbH, Germany). We then categorised species according to the salinity range of the samples the collected specimens occurred in. We first defined four salinity ranges: fresh ( $0 \% 0 \leq x \leq 2 \%$ ) , brackish ( $2 \% 0<x<25 \%$ ) , marine $(25 \%$ o $\leq \mathrm{x} \leq 50 \%$ ), and hypersaline ( $\mathrm{x}>50 \%$ ). We then assigned each species to one of 5 categories: fresh (only occurring in the fresh range), brackish (at least one sample in the brackish range, with other samples potentially in the fresh and marine range), marine (all samples in the marine range), hypersaline (at least one sample in hypersaline range, with other samples potentially in the marine range), and generalist (samples spanning the brackish, marine and hypersaline range).

We reconstructed ancestral states using stochastic character mapping (Bollback, 2006) with the R package phytools (Revell, 2012). First, we determined the most appropriate transition matrix by fitting maximumlikelihood MK-models with equal rates (ER), symmetric rates (SYM), or with all rates different (ARD) and calculated AIC weights of the models. We then performed stochastic character mapping of the best model using the Bayesian implementation with a gamma prior $(\alpha=1, \beta=1$, i.e. a prior on few transitions) and reconstructed 1000 histories (10.000 iterations burn-in followed by 10,000 iterations and retaining every 10th character history).

\section{Results and discussion}

\subsection{Phylogenetics}

\subsubsection{Large undescribed species diversity}

Based on an integrative approach-using detailed documentation of in vivo morphology and $28 S$ rRNA sequences-we identified 51 species as previously described and a striking 94 species as likely new to science (Fig. 2, Table 2), thereby increasing the species diversity in the genus by about 50\% (http://turbellaria.umaine.edu; Tyler et al., 2006). However, we need to note here, that the $28 S$ rRNA fragment used does not evolve at a fast-enough rate for the application of purely molecular based species delimitation. Therefore, it might very well be that the actual species diversity is even larger. We thus err on the side of lumping specimens, so that species with shallow molecular and no diagnostic morphological divergence are being assigned to the same operational
Table 2

Summary of the taxonomic status of all the included Macrostomum species. Species inferred based on single specimens $(\mathrm{N}=1)$ are listed separately.

\begin{tabular}{llll}
\hline Species in this study & $\mathrm{N}=1$ & $\mathrm{~N}>1$ & Total \\
\hline All & 18 & 127 & 145 \\
Described & 5 & 46 & 51 \\
Likely undescribed \& immature & 3 & 2 & 5 \\
Likely undescribed, mature \& no transcriptome & 5 & 17 & 22 \\
Likely undescribed, mature \& transcriptome & 5 & 62 & 67 \\
\hline
\end{tabular}

species. Whenever possible, we here aimed at defining species based on morphology, because this allowed us to assign specimens for which we do not have molecular data to operational taxonomic units, facilitating downstream comparative analysis (reported on separately, Brand et al., 2021)

As such, we present a first effort to organize the diversity in this extremely diverse and globally distributed genus, but we also acknowledge that further work is needed. This will have to include the application of more sophisticated molecular species delimitation methods, which will likely require the collection of more species, specimens, and molecular markers. To facilitate future taxonomic work and comparative analysis of this striking diversity, we have deposited extensive image and video data (see Brand and Schärer, 2021 for a Zenodo deposit), a vector drawing file of Fig. 2 (Fig. S1, which includes our stylet and sperm drawings), as well as geographic and molecular data for all the specimens collected (see Table S1 and S2; with each specimen carrying a unique Macrostomorpha Taxonomy and Phylogeny ID number taking the form of "MTP LS \#\#"; see also http://macrostomo rpha.info).

\subsubsection{Phylogenetic inference is robust to the methods applied}

The grouping of the major clades was mostly consistent across all phylogenetic approaches (Fig. 3 and Fig. S2). All phylogenies recovered seven large species groups (further called the hypodermic [further divided into hypodermic I and II], spirale, lignano, finnlandense, tuba, and tanganyika clade), two smaller species groups (the minutum and hamatum clade), and two consistent species pairs (M. sp. $45+46$ and $M$. sp. $4+89)$. However, the backbone, and the positions of some species with long branches (M. sp. 37, M. sp. 39, M. sp. 90 and $M$. curvituba), differed depending on the alignment and method used. Despite these discrepancies, the Robinson-Foulds distances (Robinson and Foulds, 1981) between the phylogenies were low (Table S3), indicating good agreement between the methods.

In all phylogenies, the hypodermic clade was deeply split from the reciprocal clade, but with large phylogenetic distances also appearing within the hypodermic clade (referred to as hypodermic I and II) (Fig. 3 and Fig. S2). Within the reciprocal clade the tanganyika, tuba and finnlandense clades were consistently grouped and the hamatum and minutum clades, together with $M$. sp. $4+89$, were always the closest relatives to the former three clades. However, the exact relatedness patterns were uncertain. In the L-IQ-TREE, H-IQ-TREE and H-ExaBayes phylogenies, $M$. sp. $4+89$ were the closest relatives to these three clades, followed by the hamatum and minutum clades. In contrast, in the ASTRAL phylogenies, the hamatum clade was more closely related to the minutum clade and both were sister to the grouping of tanganyika, tuba and finnlandense. Moreover, the exact branching order at the base of the reciprocal clade was not clearly resolved. The spirale clade split off first in the H-IQ-TREE phylogeny, while the lignano clade split off first in the other phylogenies.

The topology of C-IQ-TREE (Fig. 2) was identical to H-IQ-TREE (Fig. 3A and Fig. S2A) when we removed all the species added based on only $28 S$ rRNA and thus adding these species did not negatively influence the overall topology of the tree. Node support in C-IQ-TREE was somewhat lower, as could be expected given the placement of the additional species based solely on $28 S$ rRNA sequences. Nevertheless, we 

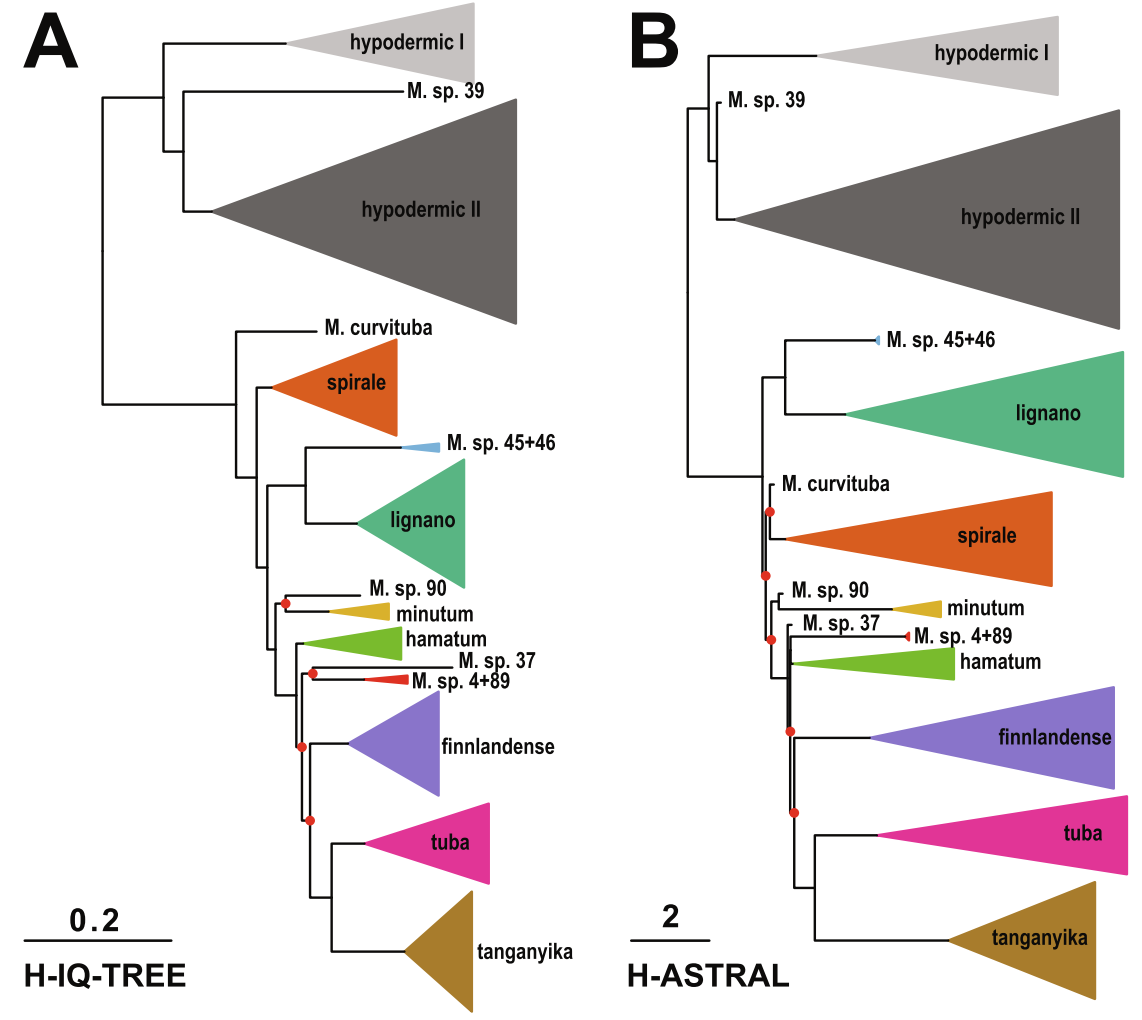
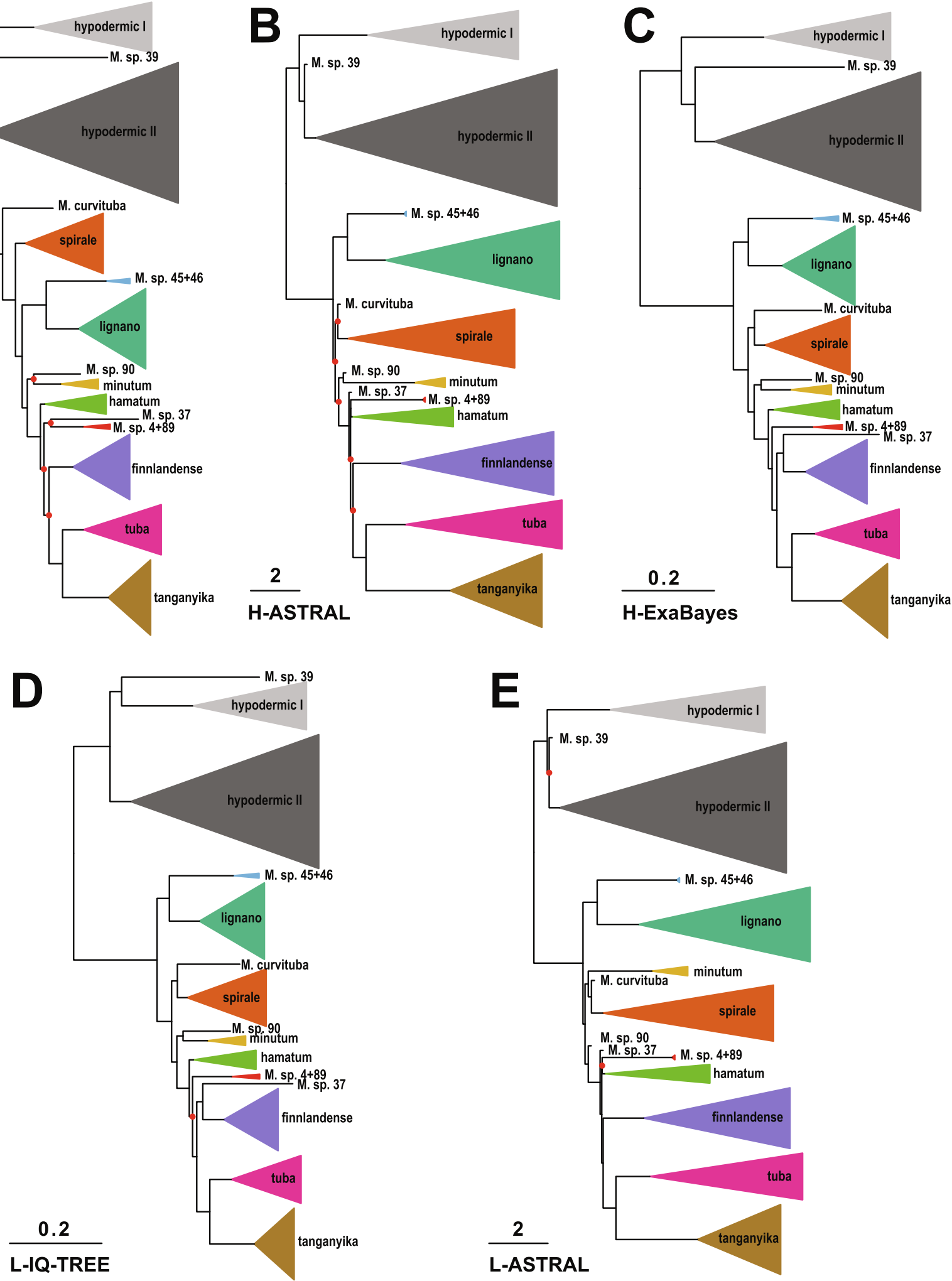

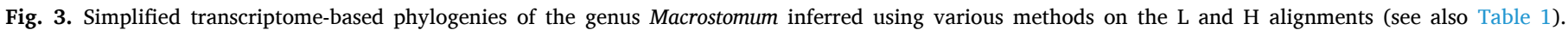

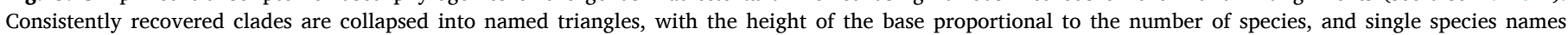

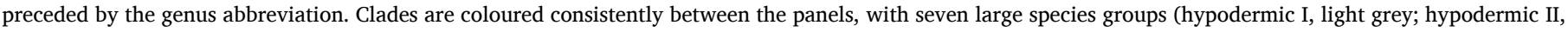

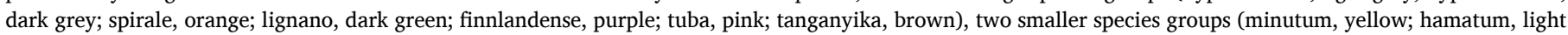

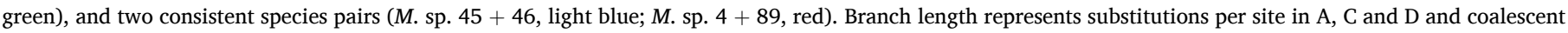

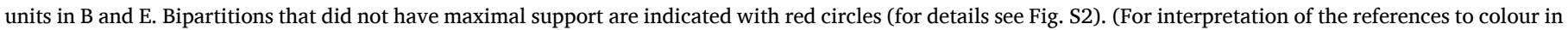
this figure legend, the reader is referred to the web version of this article.) 
think that a $\sim 50 \%$ increase in species representation is highly worthwhile, and we focus on this combined C-IQ-TREE phylogeny in the following.

Across the different phylogenies, all species represented by more than one transcriptome (i.e. $15 \times 2,3 \times 3$, and $1 \times 9$ transcriptomes) were monophyletic (Fig. S2), with the notable exception of M. lignano. Here it is interesting to point out that one M. lignano transcriptome was from the DV1 inbred line from the type locality near Bibione, Northern Adriatic Sea, Italy (Vellnow et al., 2017), while the other was from an outbred population from the Sithonia Peninsula, Aegean Sia, Greece (Zadesenets et al., 2016; Schärer et al., 2020). M. lignano was monophyletic in the L-IQ-TREE, and both ASTRAL phylogenies, but the Greek population was sister to $M$. janickei, from near Montpellier, France, in the H-IQ-TREE and H-ExaBayes phylogenies, although node support for this split was low for H-IQ-TREE (see dark green clade in Fig. S2). Moreover, removal of cDNA synthesis primer sequences from the alignment rendered $M$. lignano monophyletic also in H-IQ-TREE (see SI Primer removal, Fig. A4). A closer investigation of the morphology of the Greek vs. Italian population also revealed that the former had a considerably larger stylet ( 96.7 vs. $60.7 \mu \mathrm{m}$ ) and longer sperm (76.5 vs. $62.7 \mu \mathrm{m}$ ) (data not shown). So while we still consider $M$. lignano a proper species here, a closer comparison of these Greek and Italian populations would be interesting, as well as a broader sampling of $M$. janickei.

\subsubsection{Substantial gene tree - species tree conflict}

Consistent with the conflict between methods, the split support from the ASTRAL analysis indicated gene tree - species tree conflict at most nodes in the phylogeny's backbone (Fig. S3). These internal nodes were separated by short branches suggestive of rapid speciation events, such as during adaptive radiation (Irisarri et al., 2018), where substantial incomplete lineage sorting is expected. The pattern is also consistent with ancient and/or ongoing hybridization. Widespread hybridization is a distinct possibility, since there is evidence for hybridization between the sibling species $M$. lignano from the Eastern Mediterranean and $M$. janickei from the Western Mediterranean under laboratory conditions (Singh et al., 2020). This ability to hybridize could also explain why M. lignano was not monophyletic in some analyses (and so could a recently identified whole genome duplication, see below). Moreover, it could be interesting to explore whether the hypodermically inseminating species hybridize more frequently, since sperm injections might often be unilateral and might thus occur without active participation of the recipient, so possibly facilitating cross-species interactions. Another source of conflict could be due to whole genome duplication (WGD), leading to misidentification of ohnologs (paralogs arisen from WGD) as orthologs. This then leads to comparison of genes that do not reflect speciation, a problem compounded by differential gene loss (Kuzniar et al., 2008; Tekaia, 2016). It has been shown that M. lignano and $M$. janickei share a WGD, likely due to autotetraploidization (Zadesenets et al., 2017). Similarly, in the same clade, M. mirumnovem is also tetraploid, but it is not clear if the WGD is of the same origin, since the more closely related $M$. cliftonense shows no sign of WGD (Zadesenets et al., 2020). Clearly, WGD could be more widespread in Macrostomum, leading to gene tree - species tree conflict stems from erroneous orthology inference. To test this, more investigation based on genome sequencing would be desirable.

\subsubsection{Cryptic diversity in the hypodermic clade}

As already mentioned above, many species in the hypodermic clade were highly molecularly diverged, even though they are difficult to distinguish morphologically. Most of these species had stylets that consisted of a short proximal funnel that tapered to a curved and drawnout asymmetrical needle-like thickening (Fig. 2A, top). It was possible to distinguish some species based on general habitus, and these differences are reflected by the four deeply split clades containing, $M$. rubrocinctum, M. hystricinum, M. gabriellae, and M. pusillum, respectively (although the latter two clades were also quite similar in habitus). We have indicated substantial morphological similarity by appending a letter to the species name (e.g. M. hystricinum $a, c, d$ ). Investigations of species within the hypodermic clade without support from molecular data thus require considerable caution, and earlier authors have already identified considerable difficulties in distinguishing species with this morphology (e.g. Beklemischev, 1951; Luther, 1960; Rieger, 1977; Ladurner et al., 1997), which is even more poignant due the considerable levels of convergent evolution in the genus resulting from evolutionary shifts to hypodermic insemination (Schärer et al., 2011), which we analyse in more detail in a separate study (Brand et al., 2021). Moreover, given these striking morphological similarities, it may often not be clear to which of these species the name-bearing type specimens belong to (Schärer et al., 2011), so the species names in this clade should be considered tentative. Eventually, one may need to name these species afresh, applying more extensive molecular species delimitation, and either suppress the original names of species without detailed enough morphological descriptions and/or lacking adequate type material, or to define neotypes, including molecular voucher specimens (Pleijel et al., 2008).

\subsection{Morphological diversity}

\subsubsection{A novel stylet morphology in the hypodermic clade}

While the hypodermic clade showed little variation in terms of stylet and sperm morphology, the stylet of $M$. sp. 93 (Fig. 2A top) differed clearly from that stereotypical form, by having a small proximal funnel extending into a straight and obliquely-cut tube (Fig. 4A). This shape is similar to the stylets of several species in the reciprocal clade, namely M. shenda, M. sp. 34 (both Fig. 2A bottom) and M. sp. 64 (Fig. 2B bottom), as well as $M$. orthostylum (for which we have no phylogenetic placement). As we will report in more detail in a separate study (Brand et al., 2021), we observed hypodermic received sperm in both $M$. sp. 93 and $M$. sp. 64, and this stylet shape thus appears adapted for hypodermic insemination. While we did not observe hypodermic received sperm in M. orthostylum or M. sp. 34 (nor was such sperm reported for $M$. shenda by Xin et al., 2019), these species are nevertheless likely to also mate through hypodermic insemination, not least since stylets with similar shapes are also used for hypodermic insemination in related macrostomid flatworms (Janssen et al., 2015).

\subsubsection{Diversification of stylet and sperm morphology}

In contrast to the largely canalized stylet and sperm morphology of the hypodermic clade, we found remarkable variation in these structures within the reciprocal clade (Fig. 2). For example, we documented five species in the spirale clade that had stylets with lateral protrusions close to the distal opening (Fig. 2A middle). The protrusion is shaped like a rod or spike in M. evelinae, $M$. sp. 29 and $M$. sp. 42, while it consists of two thin filaments in M. sp. 30 and M. sp. 43 (Fig. 4B; see also Fig. A13 in SI Species delimitation). These highly modified stylets may have coevolved with the complex antra in these species, which have two separate chambers connected via a ciliated muscular sphincter. The sperm of these species is also remarkable because they carry only a single bristle instead of the typical two. The sperm of $M$. sp. 30 carry only a single curved bristle, while the bristle of $M$. evelinae, $M$. sp. 29, and M. sp. 42 (Fig. 4D) is additionally modified, being thicker and appearing flexible, frequently curving back towards the sperm body. A second bristle might also be absent in the closely related $M$. sp. 13 (indicated with a shaded second bristle in Fig. 2A middle), but the available material currently does not allow an unambiguous assessment.

Sperm are also highly variable across the entire reciprocal clade. Particularly striking are the sperm modifications of $M$. sp. 82 (Fig. 2B top), which give the anterior part of the sperm a translucent appearance under phase contrast (Fig. 4E), and which provides motility that is different from that observed in normal sperm feelers. We also document extraordinarily long sperm in several species in the tanganyika clade (Fig. 2B bottom). While it is not entirely clear which part of the sperm is 

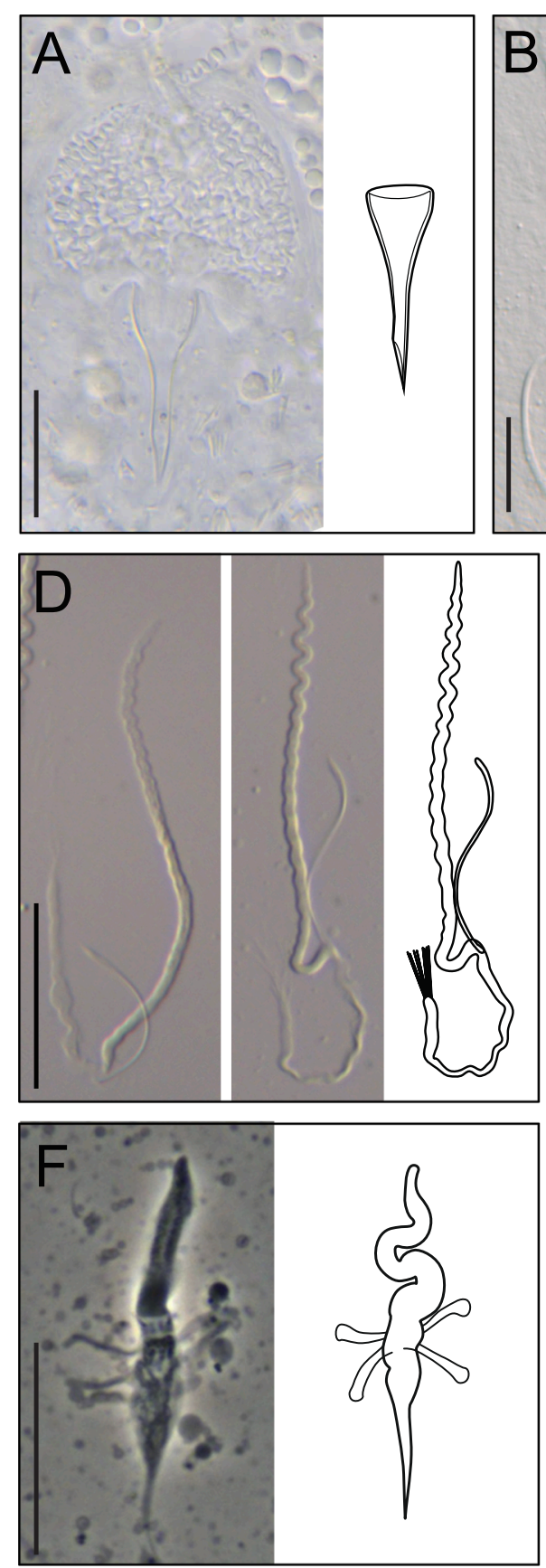
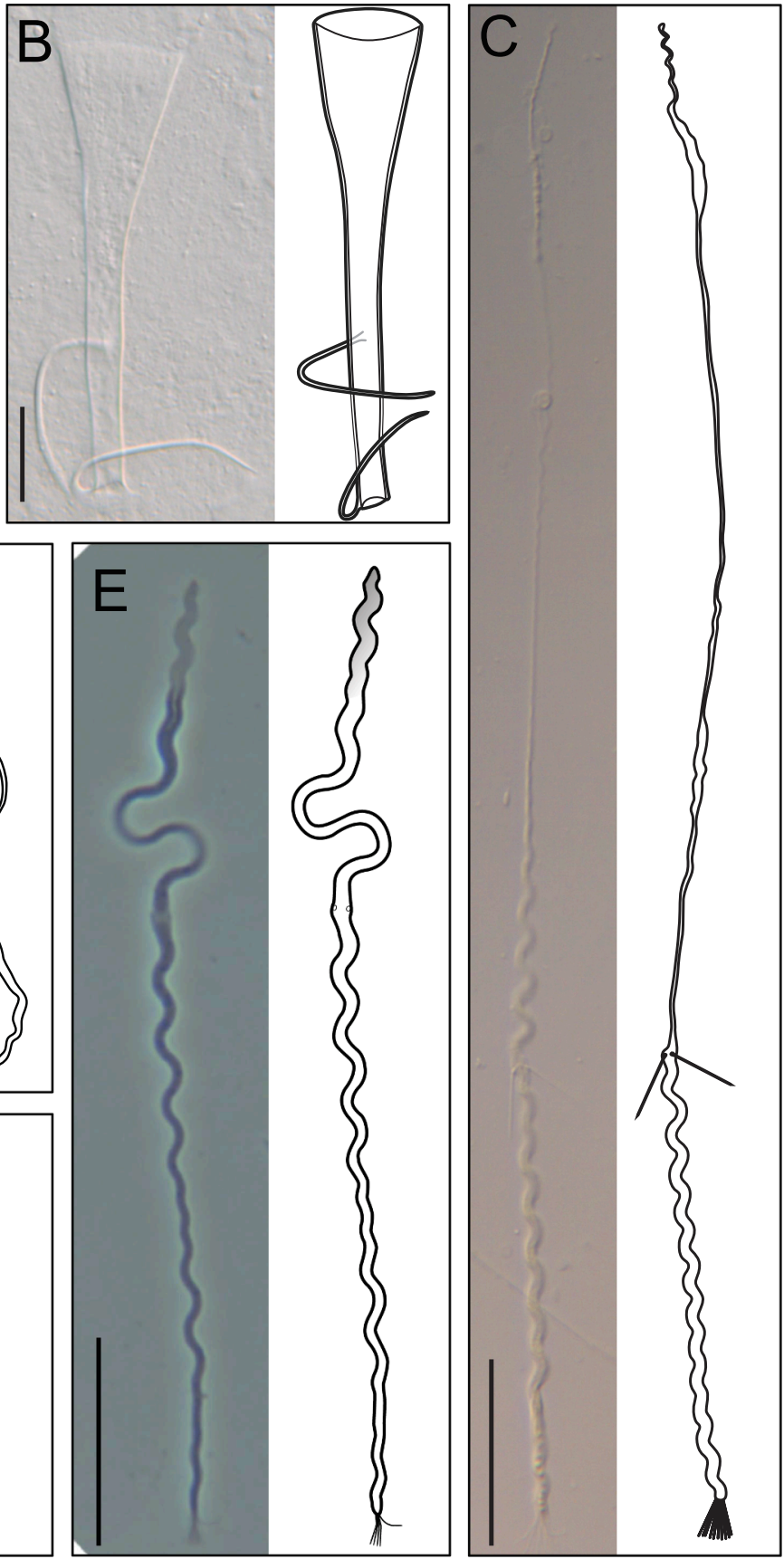

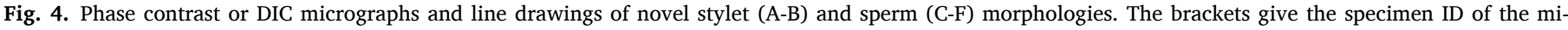

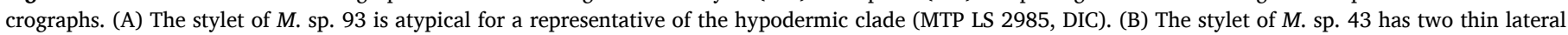

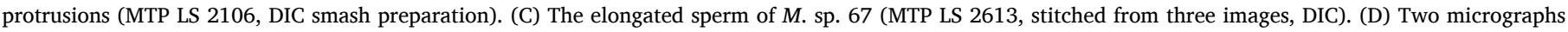

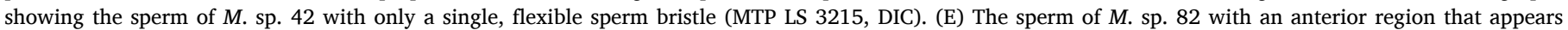

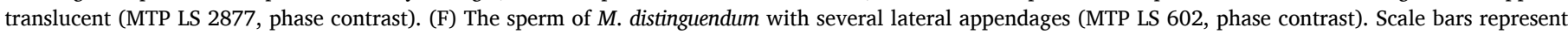
$20 \mu \mathrm{m}$.

modified here, it appears that they have very long sperm feelers, with the bristles thus being located unusually far posterior, as in M. sp. 67 (Fig. 4C). These long feelers represent a novel character in this African clade and should be interesting targets of future investigations. Additionally, we observed numerous species that had short or no sperm bristles across the whole reciprocal clade and appeared to coincide with changes in the stylet morphology. But as already mentioned above, a more formal analysis of convergent evolution in stylet and sperm morphology, including such reductions and losses of sperm bristles, will be presented in a separate study (Brand et al., 2021). Finally, a striking modification of sperm design occurs in $M$. distinguendum in the finnlandense clade (Fig. 2B middle), which appears to lack sperm bristles, but instead carries novel club-shaped lateral appendages, which in light microscopic images bear no resemblance to the usual sperm bristles (Fig. 4F).

\subsection{Ancestral state reconstruction}

The ancestral state reconstruction of the habitat salinity indicated that the most recent common ancestor was either marine or brackish ( $58 \%$ and $38 \%$ of reconstructions, respectively), which matches the habitat of the outgroup, Psammomacrostomum. Marine and brackish 
species appear well mixed and within these clades several species also occur in hypersaline habitats, and a few species appear to be generalists (Fig. 5, Tab. S4). Freshwater species show strong phylogenetic clustering. Two freshwater species ( $M$. sp. 34 and $M$. sp. 36) do not cluster with the others, potentially indicating that the invasion of freshwater may have occurred more than once. However, it is also possible that these species are simply brackish species collected at salinities below our detection limit. In summary, the reconstruction clearly indicates that, although many Macrostomum have a wide salinity range, they do not frequently transition to complete freshwater environments. This is potentially also interesting from a biogeographic perspective, since Macrostomum have been collected in freshwater on all continents (except Antarctica; http://turbellaria.umaine.edu).

\subsection{Taxonomic implications}

The large-scale collections and molecular phylogenetic analyses we present here have a number of implications for the taxonomy and nomenclature of the Macrostomidae (Tyler et al., 2006), and they support several taxonomic and nomenclatural revisions. We outline these

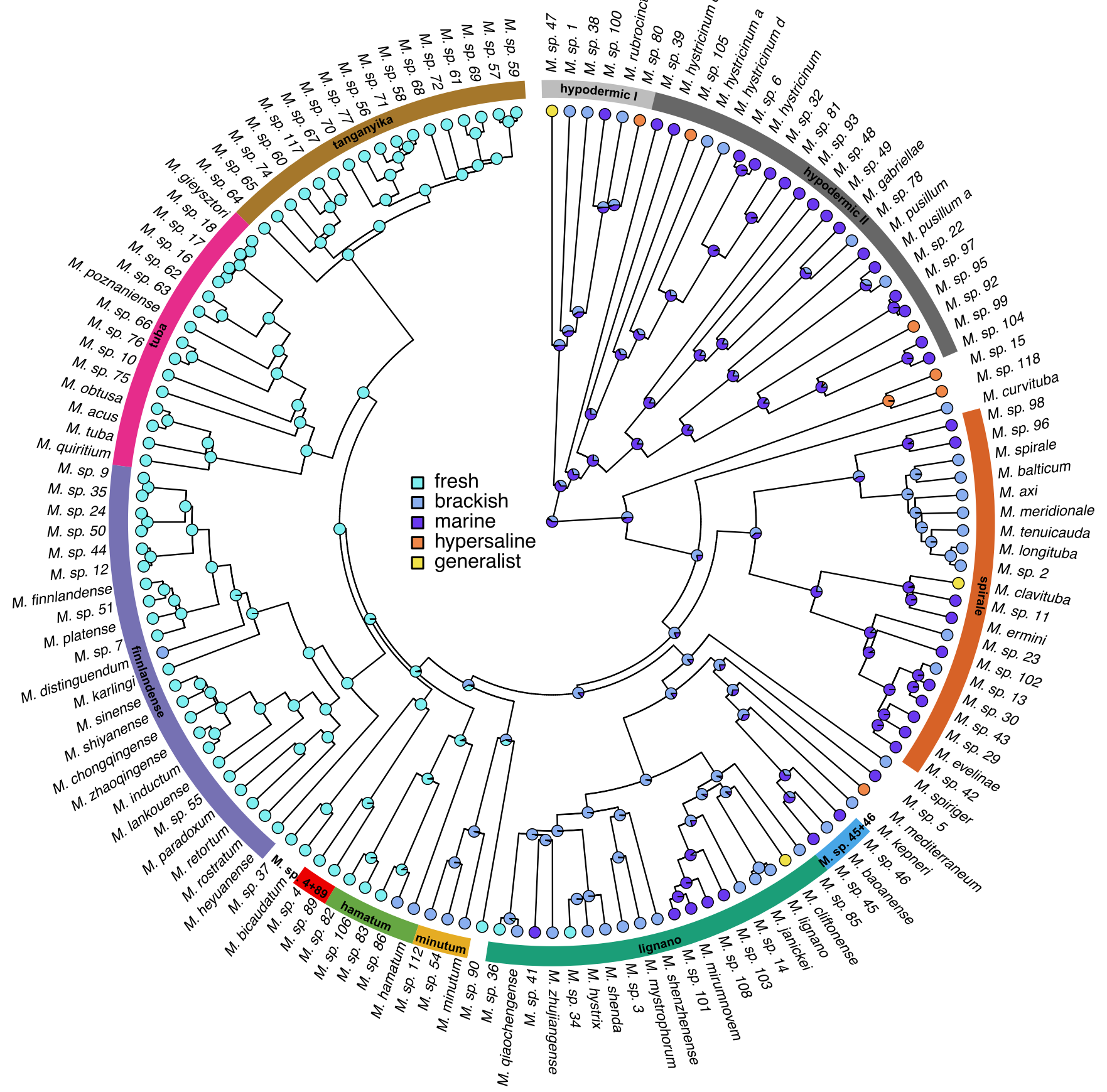

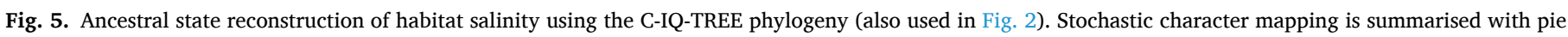

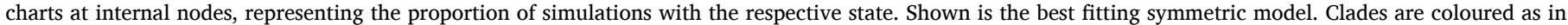

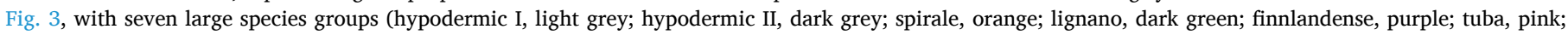

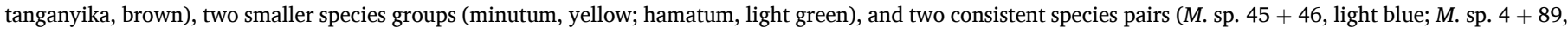

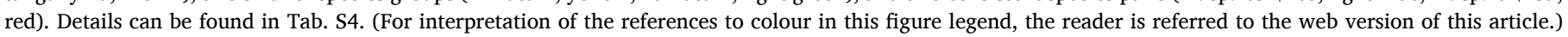


changes in the following, using the "Genus species Author, Year" format to refer to binomials, and additionally including all the citations for the relevant works.

\subsubsection{Bradburia is questionable}

As part of the description of some Australian macrostomids, Faubel et al. (1994) established the genus Bradburia Faubel, Blome \& Cannon, 1994 for species with a "Male system with accessory stylet connected to tubular stylet proximally" as the most prominent diagnostic trait. They named a new species, Bradburia australiensis Faubel, Blome \& Cannon, 1994, and transferred a species originally described as Macrostomum miraculicis Schmidt \& Sopott-Ehlers, 1976 (Schmidt and Sopott-Ehlers, 1976) to this genus, designating it Bradburia miraculicis (Schmidt \& Sopott-Ehlers, 1976) and making it the type species of the genus Bradburia.

While we have collected neither of these two species ourselves, we have identified several species that carry some kind of lateral protrusion on the stylet shaft. These include Macrostomum inductum Kolasa, 1971 (Kolasa, 1971) and Macrostomum sp. 108 (Fig. 2B middle and Fig. 2A bottom, respectively), which have independently evolved ridge-like protrusions on the distal stylet. In addition, we have recovered a clade that includes Macrostomum evelinae Marcus, 1946 (Marcus, 1946), in which all but one species carries elongated lateral protrusions on the stylet shaft, albeit not proximally, but rather in the distal third of the stylet (Fig. 2A middle). Note that also in B. miraculicis the lateral protrusion is not actually positioned proximally on the stylet-as the diagnosis of the genus Bradburia would imply_but according to Schmidt \& Sopott-Ehlers (Schmidt and Sopott-Ehlers, 1976) it emerges about half-way ("Etwa auf halber Länge ..."). Moreover, Schmidt \& Sopott-Ehlers draw that lateral protrusion as an integral part of the stylet, rather than an accessory stylet (as is, for example, observed in the Dolichomacrostomids), which therefore does not conform to the Bradburia genus diagnosis. In contrast, the lateral protrusion of the stylet of B. australiensis, as drawn by Faubel et al., could possibly be somewhat more independent.

Other diagnostic traits of the genus Bradburia are also not decisive, including the absence of eyes (a widespread trait in the genus Macrostomum, including the above-mentioned $M$. evelinae clade) and the presence of cuticularised parts in the female antrum, which are, for example, also observed in Macrostomum ermini Ax, 1959 (Fig. 2A middle) (Ax, 1959, a species that lacks lateral stylet protrusions). Surprisingly, the organization of the female system was not observed in the description of $B$. australiensis, which raises the question why Faubel et al. (1994) consider it Bradburia.

The above considerations suggest that the genus Bradburia is currently not well-supported and we think it likely that molecular analyses would lead to the placement of both $B$. miraculicis and B. australiensis in the reciprocal clade of the genus Macrostomum, and possibly even into the M. evelinae clade, which may thus lead to the eventual suppression of the genus Bradburia.

\subsubsection{Promacrostomum should be dropped}

Based on specimens collected from Lake Ohrid, An-der-Lan (1939) established a new genus Promacrostomum An-der-Lan, 1939, with initially a single representative, Promacrostomum paradoxum An-derLan, 1939 based on the presence of two female genital openings and a genito-intestinal duct. In all our phylogenies An-der-Lan's species was placed within the reciprocal clade as sister to M. retortum Papi, 1951 (Papi, 1951). Moreover, a second female opening has independently evolved multiple times in the genus Macrostomum (Schärer et al., 2011; Xin et al., 2019), so this trait is homoplastic, and it alone is clearly not a useful synapomorphy for this genus. We, therefore, transfer this species from Promacrostomum to Macrostomum paradoxum (An-der-Lan, 1939). Moreover, as outlined by Schärer et al. (2011), another species with two female genital openings, Macrostomum gieysztori Ferguson, 1939 (Ferguson, 1939), also belongs to Macrostomum, despite it having previously been placed into Promacrostomum by Papi (Papi, 1950, 1951) and later the genus Axia by Ferguson (1954).

With this reclassification, the genus Promacrostomum loses the type species and would only contain Promacrostomum palum Sluys, 1986 (Sluys, 1986), which was assigned to this genus because it, like M. paradoxum, has two female genital openings (while a genitointestinal duct was not observed by Sluys). While it seems likely that a molecular placement of this species would reveal that it belongs to Macrostomum, it is unclear whether it would group with any of the known representatives with this trait state or represent yet an additional independent origin. Given that the main characteristic of the genus Promacrostomum, namely the presence of two female genital openings, has several independent origins, we consider Promacrostomum unjustified and transfer its last remaining species from Promacrostomum to Macrostomum palum (Sluys, 1986).

\subsection{3. $M$. zhaoqingense could be a re-description of $M$. inductum}

The comparison of $28 S$ rRNA sequences has revealed that Macrostomum zhaoqingense Lin \& Wang, 2017 (Lin et al., 2017b) is sister to Macrostomum inductum Kolasa, 1971 (Kolasa, 1971) and the haplotype analysis closely clusters both species (see Fig. A19 in SI Species delimitation), suggesting that $M$. zhaoqingense could potentially represent a redescription of $M$. inductum. More detailed molecular and morphological comparisons are called for to determine the taxonomic status of M. zhaoqingense.

\subsubsection{Macrostomum meridionale is raised to species}

Based primarily on affinities in the stylet morphology and the female genital morphology, Papi (1953) described Macrostomum balticum meridionalis Papi, 1953 as a subspecies of M. balticum Luther, 1947. M. balticum meridionalis was described from brackish water in the San Rossore park near Pisa, Italy (Papi, 1953), and M. balticum from the Baltic Sea in the Gulf of Finland (Luther, 1947). M. balticum has since also been found in the Skagerrak and the North Sea (Luther, 1960; Schärer et al., 2011; this paper). Our phylogenetic placement of a specimen of M. balticum meridionalis (MTP LS 321)—collected close to the type locality in the San Rossore park-identifies it as a member of the spirale clade. While the spirale clade also contains $M$. balticum, our specimen is clearly genetically diverged from it and any other species in that clade. We, therefore, raise this subspecies to species level, and designate it Macrostomum meridionale Papi, 1953. We thereby adjust the ending of the species name to reflect the fact that the genus name Macrostomum is neuter (as recently pointed out by Zhang et al. (2021); this also affects nearly twenty other names in the genus and we use these updated names here).

\subsection{Conclusions}

Our detailed phylogenomic investigations of 145 Macrostomum species show that the genus consists of two well-separated clades. Across phylogenetic methods the grouping within the, morphologically canalized, hypodermic clade is robust, but the exact interrelationships within the, morphologically diverse, reciprocal clade are somewhat less clear. However, we also find highly consistent subclades within the reciprocal clade, with most conflicts between methods stemming from uncertainty in the backbone of the phylogeny. Short internal branches of all phylogenies, and low split support from the ASTRAL analysis, suggest that this likely occurs because of a rapid radiation at the base of the reciprocal clade. Such a radiation could then lead to substantial gene tree species tree conflict due to incomplete lineage sorting. Moreover, widespread current and/or ancient hybridization could also cause these gene tree - species tree conflicts.

Remarkably, 94 of the collected species are likely new to science, highlighting that a large proportion of the diversity in this genus is yet to be discovered. Our increased taxon sampling has not only yielded many more species, but these additional species have also revealed a range of 
novel morphological traits. While some of these traits are phylogenetically clustered, others, which have previously been considered as diagnostic synapomorphies for the erection of separate genera, are show to be homoplastic, thus requiring several taxonomic changes. The striking convergence of a range of traits makes taxonomic assignments solely based on morphology questionable and we suggest that future work, particularly on species exhibiting the hypodermic morphology, should always include molecular markers. The need for employing molecular markers is further supported by the large cryptic diversity within the hypodermic clade, a taxonomically challenging group that, however, could be interesting for investigations of cryptic speciation.

\section{CRediT authorship contribution statement}

Jeremias N. Brand: Conceptualization, Data curation, Formal analysis, Investigation, Visualization, Writing - original draft, Writing review \& editing. Gudrun Viktorin: Investigation, Methodology. R. Axel W. Wiberg: Investigation, Writing - review \& editing. Christian Beisel: Methodology, Resources. Lukas Schärer: Conceptualization, Data curation, Funding acquisition, Investigation, Project administration, Resources, Supervision, Writing - original draft, Writing - review \& editing.

\section{Declaration of Competing Interest}

The authors declare that they have no known competing financial interests or personal relationships that could have appeared to influence the work reported in this paper.

\section{Acknowledgments}

We thank the numerous people that have helped with field work. Especially, we are grateful for the help of, in no particular order, Werner Armonies, Benny Glasgow, Mohamed Charni, Edith Zemp, Bernhard Egger, Peter Ladurner, Gregor Schulte, Floriano Papi, Kazuya Kobayashi, Christopher Laumer, Wim Willems, Tom Artois, Christian Lott, Miriam Weber, Ana-Maria Leal-Zanchet, Kaja Wasik, Mariana Adami, Walter Salzburger, Adrian Indermaur, Bernd Egger, Fabrizia Ronco, Heinz Büscher, Victoria Huwiler, Philipp Kaufmann, Michaela Zwyer, Stefanie von Fumetti, Joe Ryan, Mark Q. Martindale, Marta Chiodin, John Evans, Leigh Simmons, Mauro Tognon, Piero Tognon, Cristiano Tognon, Pragya Singh, Nikolas Vellnow, Christian Felber, Ulf Jondelius, Sarah Atherton, Tim Janicke, Georgina Rivera-Ingraham, Ben Byrne, Yvonne Gilbert, Rod Watson, Jochen Rink, Miquel Vila-Fare, Helena Bilandžija, and Sasho Trajanovski. We thank Katja Eschbach of the Genomics Facility Basel for preparing and sequencing RNA-Seq libraries. We thank Peter Fields and Lukas Zimmermann for IT advice. We thank Jürgen Hottinger, Daniel Lüscher and Yasmin Picton for administrative and technical support. We thank Dita Vizoso for the use of sperm and stylet drawings for some of the previously described species and for providing inspiration for the new drawings. We thank Yu Zhang and his collaborators for kindly providing specimens of $M$. baoanense. We thank the following locations and authorities for collection/export permits, Western Australia (DPAW Reg 17 01-000135-2 and Reg 18 OS002550), Victoria (DELWP National Parks Act 1975 10008144), Tenuta di San Rossore, Italy (3299/7-2-1/2010 and 1337/7-2-1/2016), Parco Nazionale Arcipelago Toscano, Italy (4440/2011), Sao Leopoldo, Brazil (IBAMA 12BR008859/DF), Carrie Bow Cay, Belize (STRI Field Station and Department of Fisheries), Lakes Ohrid and Prespa, North Macedonia (Hydrobiological Institute Ohrid), Swedish West Coast (Kristineberg Biological Station), Reserve Naturelle de la Petite Camargue Alsacienne, France (years 2016 and 2017), Florida, USA (Whitney Laboratory for Marine Bioscience), Sardinia, Italy (Regione Autonoma della Sardegna 0035419/2018), Zambia (University of Zambia in Lusaka, Department of Fisheries, Department of Immigration, and KA/B/29/16), Malawi, (Museum Karonga and Fisheries Department).We thank Nick Goldman and Ziheng Yang for organizing the summer school on Computational Molecular Evolution, which has helped and inspired the first author to explore phylogenetics. Calculations were performed, in part, at sciCORE (http://scicore.unibas.ch/) scientific computing centre at the University of Basel.

\section{Funding}

This work was supported by Swiss National Science Foundation (SNSF) research grants 31003A_162543 and 310030_184916 to LS.

\section{Data availability}

The raw sequencing data generated for this study are available in the NCBI Sequence Read Archive repository with the following accession: PRJNA635941. The partial 28S rRNA sequence are available on NCBI with the following accessions: MT428556-MT429159. Extensive image and video material of all documented specimens are deposited on Zenodo at: 10.5281/zenodo.4482135. Transcriptome assemblies, gene alignments and phylogenetic trees are deposited on Zenodo at: 10.5281/ zenodo.4543289.

\section{Appendix A. Supplementary data}

Supplementary data to this article can be found online at https://doi. org/10.1016/j.ympev.2021.107296.

\section{References}

Aberer, A.J., Kobert, K., Stamatakis, A., 2014. Exabayes: massively parallel Bayesian tree inference for the whole-genome era. Mol. Biol. Evol. 31, 2553-2556.

Adami, M., Damborenea, C., Ronderos, J.R., 2012. A new limnic species of Macrostomum (Platyhelminthes: Macrostomida) from Argentina and its muscle arrangement labeled with phalloidin. Zool. Anz. - J. Comp. Zool. 251 (3), 197-205.

An-der-Lan, H., 1939. Zur rhabdocoelen Turbellarienfauna des Ochridasees (Balkan). Sitzungsberichte Mathem-Naturw Kl Abt 148 (5), 196-254.

Atherton, S., Jondelius, U., Michaux, J.R., 2019. A taxonomic review and revisions of Microstomidae (Platyhelminthes: Macrostomorpha). PLoS ONE 14 (4), e0212073. https://doi.org/10.1371/journal.pone.0212073.

Ax, P., 1959. Zur Systematik, Ökologie und Tiergeographie der Turbellarienfauna in den ponto-kaspischen Brackwassermeeren. Zool Jb Syst. 87, 43-187.

Beklemischev, V.N., 1951. Macrostomum (Turbellaria, Rhabdocoela) of the USSR (O vidach roda Macrostomum SSSR) [In Russian]. Z. Mosk. Naturwissenschaftlichen Ges. 56, 31-40.

Bollback, J.P., 2006. SIMMAP: stochastic character mapping of discrete traits on phylogenies. BMC Bioinf. 7, 88.

Brand, J. N., Harmon, L.J., Schärer, L., 2021. Frequent origins of traumatic insemination involve convergent shifts in sperm and genital morphology. bioRxiv 2021.02.16.431427.

Brand, J.N., Schärer, L., 2021b. Specimens from Brand et al., 2021, "Large-scale phylogenomics of the genus Macrostomum (Platyhelminthes) reveals cryptic diversity and novel sexual traits". Zenodo. https://doi.org/10.5281/ zenodo.4482135.

Brand, J.N., Wiberg, R.A.W., Pjeta, R., Bertemes, P., Beisel, C., Ladurner, P., Schärer, L., 2020. RNA-Seq of three free-living flatworm species suggests rapid evolution of reproduction-related genes. BMC Genomics 21, 462.

Capella-Gutierrez, S., Silla-Martinez, J.M., Gabaldon, T., 2009. trimAl: a tool for automated alignment trimming in large-scale phylogenetic analyses. Bioinformatics 25, 1972-1973.

de Chambrier, A., Zehnder, M., Vaucher, C., Mariaux, J., 2004. The evolution of the Proteocephalidea (Platyhelminthes, Eucestoda) based on an enlarged molecular phylogeny, with comments on their uterine development. Syst. Parasitol. 57 (3), 159-171.

de Chambrier, A., Waeschenbach, A., Fisseha, M., Scholz, T., Mariaux, J., 2015. A large $28 \mathrm{~S}$ rDNA-based phylogeny confirms the limitations of established morphological characters for classification of proteocephalidean tapeworms (Platyhelminthes, Cestoda). ZooKeys 500, 25-59.

Clement, M., Posada, D., Crandall, K.A., 2000. TCS: a computer program to estimate gene genealogies. Mol. Ecol. 9, 1657-1659.

Egger, B., Ladurner, P., Nimeth, K., Gschwentner, R., Rieger, R., 2006. The regeneration capacity of the flatworm Macrostomum lignano - on repeated regeneration, rejuvenation, and the minimal size needed for regeneration. Dev. Genes Evol. 216, 565-577.

Emms, D.M., Kelly, S., 2015. OrthoFinder: solving fundamental biases in whole genome comparisons dramatically improves orthogroup inference accuracy. Genome Biol. 16, 157. 
Faubel, A., Blome, D., Cannon, L.R.G., 1994. Sandy beach meiofauna of eastern Australia (southern Queensland and New South Wales). I. Introduction and Macrostomida (Platyhelminthes). Invertebr. Syst. 8 (4), 899. https://doi.org/10.1071/IT9940989.

Ferguson, F.F., 1939. A monograph of the genus Macrostomum O. Schmidt 1848. Part IV Zool. Anz. 128, 188-205.

Ferguson, F.F., 1954. Monograph of the Macrostomine worms of turbellaria. Trans. Am. Microsc. Soc. 73 (2), 137. https://doi.org/10.2307/3223751.

Fu, L., Niu, B., Zhu, Z., Wu, S., Li, W., 2012. CD-HIT: accelerated for clustering the nextgeneration sequencing data. Bioinformatics 28, 3150-3152.

Grudniewska, M., Mouton, S., Grelling, M., Wolters, A.H.G., Kuipers, J., Giepmans, B.N. G., Berezikov, E., 2018. A novel flatworm-specific gene implicated in reproduction in Macrostomum lignano. Sci. Rep. 8, 3192.

Grudniewska, M., Mouton, S., Simanov, D., Beltman, F., Grelling, M., de Mulder, K., Arindrarto, W., Weissert, P.M., van der Elst, S., Berezikov, E., 2016. Transcriptional signatures of somatic neoblasts and germline cells in Macrostomum lignano. eLife 5.

Haas, B.J., Papanicolaou, A., Yassour, M., Grabherr, M., Blood, P.D., Bowden, J. Couger, M.B., Eccles, D., Li, B., Lieber, M., MacManes, M.D., Ott, M., Orvis, J., Pochet, N., Strozzi, F., Weeks, N., Westerman, R., William, T., Dewey, C.N., Henschel, R., LeDuc, R.D., Friedman, N., Regev, A., 2013. De novo transcript sequence reconstruction from RNA-seq using the Trinity platform for reference generation and analysis. Nat. Protoc. 8 (8), 1494-1512.

Irisarri, I., Singh, P., Koblmüller, S., Torres-Dowdall, J., Henning, F., Franchini, P., Fischer, C., Lemmon, A.R., Lemmon, E.M., Thallinger, G.G., Sturmbauer, C., Meyer, A., 2018. Phylogenomics uncovers early hybridization and adaptive loci shaping the radiation of Lake Tanganyika cichlid fishes. Nat. Commun. 9, 3159.

Janicke, T., Schärer, L., 2010. Sperm competition affects sex allocation but not sperm morphology in a flatworm. Behav. Ecol. Sociobiol. 64 (9), 1367-1375.

Janssen, T., Vizoso, D.B., Schulte, G., Littlewood, D.T.J., Waeschenbach, A., Schärer, L., 2015. The first multi-gene phylogeny of the Macrostomorpha sheds light on the evolution of sexual and asexual reproduction in basal Platyhelminthes. Mol. Phylogenet. Evol. 92, 82-107.

Kalyaanamoorthy, S., Minh, B.Q., Wong, T.K.F., von Haeseler, A., Jermiin, L.S., 2017. ModelFinder: fast model selection for accurate phylogenetic estimates. Nat. Methods 14 (6), 587-589.

Kolasa, J., 1971. Two new species of microturbellaria of the genera Stenostomum O. Schmidt and Macrostomum O. Schmidt. Bull. Académie Pol. Sci. Sér. Sci. Biol. 19, 743-747.

Kuzniar, A., van Ham, R.C.H.J., Pongor, S., Leunissen, J.A.M., 2008. The quest for orthologs: finding the corresponding gene across genomes. Trends Genet. 24 (11), 539-551.

Ladurner, P., Mair, G.R., Reiter, D., Salvenmoser, W., Rieger, R.M., 1997. Serotonergic nervous system of two Macrostomid species: recent or ancient divergence? Invertebr. Biol. 116, 178 .

Ladurner, P., Schärer, L., Salvenmoser, W., Rieger, R.M., 2005. A new model organism among the lower Bilateria and the use of digital microscopy in taxonomy of meiobenthic Platyhelminthes: Macrostomum lignano, n. sp. (Rhabditophora, Macrostomorpha). J. Zool. Syst. Evol. Res. 43, 114-126.

Laumer, C.E., Hejnol, A., Giribet, G., 2015. Nuclear genomic signals of the 'microturbellarian' roots of platyhelminth evolutionary innovation. eLife 4.

Lengerer, B., Hennebert, E., Flammang, P., Salvenmoser, W., Ladurner, P., 2016. Adhesive organ regeneration in Macrostomum lignano. BMC Dev. Biol. 16, 20.

Lin, Y., Zhou, W., Xiao, P., Zheng, Y., Lu, J., Li, J., Wang, A., 2017a. Two new species of freshwater Macrostomum (Rhabditophora: Macrostomorpha) found in China. Zootaxa 4329 (3), 267. https://doi.org/10.11646/zootaxa.4329.310.11646/ zootaxa.4329.3.5.

Lin, Y.-T., Feng, W.-T., Xin, F., Zhang, L., Zhang, Y.U., Wang, A.-T., 2017b. Two new species and the molecular phylogeny of eight species of Macrostomum (Platyhelminthes: Macrostomorpha) from southern China. Zootaxa 4337 (3), 423. https://doi.org/10.11646/zootaxa.4337.310.11646/zootaxa.4337.3.7.

Luther, A., 1960. Die Turbellarien Ostfennoskandiens I. Acoela, Catenulida, Macrostomida, Lecithoepitheliata, Prolecithophora, und Proseriata. Fauna Fenn. 7, $1-155$.

Luther, A., 1947. Untersuchungen an rhabdocoelen Turbellarien VI. Macrostomiden aus Finnland. Acta Zool. Fenn. 49, 1-38.

Marcus, E., 1946. Sôbre Turbellaria brasileiros. Bol Fac Fil Ciênc Letr Univ São Paulo Zool 11 (11), 5. https://doi.org/10.11606/issn.2526-4877. bsffclzoologia.1946.125301.

Marie-Orleach, L., Vellnow, N., Schärer, L., 2021. The repeatable opportunity for selection differs between pre- and postcopulatory fitness components. Evol. Lett. 5 (1), 101-114.

Mouton, S., Grudniewska, M., Glazenburg, L., Guryev, V., Berezikov, E., 2018. Resilience to aging in the regeneration-capable flatworm Macrostomum lignano. Aging Cell 17 (3), e12739. https://doi.org/10.1111/acel.2018.17.issue-310.1111/acel.12739.

Nakamura, T., Yamada, K.D., Tomii, K., Katoh, K., Hancock, J., 2018. Parallelization of MAFFT for large-scale multiple sequence alignments. Bioinformatics 34, 2490-2492.

Nguyen, L.-T., Schmidt, H.A., von Haeseler, A., Minh, B.Q., 2015. IQ-TREE: a fast and effective stochastic algorithm for estimating maximum-likelihood phylogenies. Mol. Biol. Evol. 32, 268-274.

Papi, F., 1951. Richerche sui Turbellari Macrostomidae. Arch. Zool. Ital. 36, 289-341.

Papi, F., 1950. Sulle affinità morfologiche nella fam. Macrostomidae (Turbellaria). Bolletino Zool. 17 (sup002), 461-468.

Papi, F., 1953. Beiträge zur Kenntnis der Macrostomiden (Turbellarien). Acta Zoologica Fennica 78, 1-32.

Pfister, D., De Mulder, K., Hartenstein, V., Kuales, G., Borgonie, G., Marx, F., Morris, J., Ladurner, P., 2008. Flatworm stem cells and the germ line: developmental and evolutionary implications of macvasa expression in Macrostomum lignano. Dev. Biol. 319 (1), 146-159.

Pfister, D., De Mulder, K., Philipp, I., Kuales, G., Hrouda, M., Eichberger, P., Borgonie, G., Hartenstein, V., Ladurner, P., 2007. The exceptional stem cell system of Macrostomum lignano: Screening for gene expression and studying cell proliferation by hydroxyurea treatment and irradiation. Front. Zool. 4 (1), 9. https://doi.org/ 10.1186/1742-9994-4-9.

Pleijel, F., Jondelius, U., Norlinder, E., Nygren, A., Oxelman, B., Schander, C., Sundberg, P., Thollesson, M., 2008. Phylogenies without roots? A plea for the use of vouchers in molecular phylogenetic studies. Mol. Phylogenet. Evol. 48 (1), 369-371.

Plummer, M., Best, N., Cowles, K., Vines, K., 2006. CODA: Convergence diagnosis and output analysis for MCMC. R News 6, 7-11.

Revell, L.J., 2012. phytools: an R package for phylogenetic comparative biology (and other things): phytools: R package. Methods Ecol. Evol. 3, 217-223.

Rieger, R.M., 1977. The relationship of character variability and morphological complexity in copulatory structures of Turbellaria-Macrostomida and -Haplopharyngida. Mikrofauna Meeresbod. 61, 197-216.

Robinson, D.F., Foulds, L.R., 1981. Comparison of phylogenetic trees. Math. Biosci. 53 (1-2), 131-147.

Roure, B., Baurain, D., Philippe, H., 2013. Impact of missing data on phylogenies inferred from empirical phylogenomic data sets. Mol. Biol. Evol. 30, 197-214.

Sanderson, M.J., 2002. Estimating absolute rates of molecular evolution and divergence times: a penalized likelihood approach. Mol. Biol. Evol. 19, 101-109.

Scarpa, F., Cossu, P., Sanna, D., Lai, T., Norenburg, J.L., Curini-Galletti, M., Casu, M., 2015. An 18S and 28S-based clock calibration for marine Proseriata (Platyhelminthes). J. Exp. Mar. Biol. Ecol. 463, 22-31.

Schärer, L., Brand, J.N., Singh, P., Zadesenets, K.S., Stelzer, C.-P., Viktorin, G., 2020. A phylogenetically informed search for an alternative Macrostomum model species, with notes on taxonomy, mating behavior, karyology, and genome size. J. Zool. Syst. Evol. Res. 58 (1), 41-65.

Schärer, L., Joss, G., Sandner, P., 2004. Mating behaviour of the marine turbellarian Macrostomum sp.: these worms suck. Mar. Biol. 145, 373-380.

Schärer, L., Littlewood, D.T.J., Waeschenbach, A., Yoshida, W., Vizoso, D.B., 2011. Mating behavior and the evolution of sperm design. Proc. Natl. Acad. Sci. 108, 1490-1495.

Schmidt, P., Sopott-Ehlers, B., 1976. Interstitielle Fauna von Galapagos XV. Macrostomum o. Schmidt, 1848 und Siccomacrostomum triviale nov. gen. nov. spec. (Turbellaria, Macrostomida). Mikrofauna Meeresbod. 57, 1-45.

Simmons, M.P., Müller, K., Norton, A.P., 2007. The relative performance of indel-coding methods in simulations. Mol. Phylogenet. Evol. 44 (2), 724-740.

Singh, P., Ballmer, D.N., Laubscher, M., Schärer, L., 2020. Successful mating and hybridisation in two closely related flatworm species despite significant differences in reproductive morphology and behaviour. Sci. Rep. 10, 12830.

Sluys, R., 1986. First representative of the order Macrostomida in Australia (Platyhelminthes, Macrostomidae). Rec. South Aust. Mus. 19, 399-404.

Smith, S.A., O'Meara, B.C., 2012. treePL: Divergence time estimation using penalized likelihood for large phylogenies. Bioinformatics 28, 2689-2690.

Smith-Unna, R., Boursnell, C., Patro, R., Hibberd, J.M., Kelly, S., 2016. TransRate: reference-free quality assessment of de novo transcriptome assemblies. Genome Res. 26 (8), 1134-1144.

Sun, T., Zhang, L., Wang, A.-T., Zhang, Y., 2015. Three new species of freshwater Macrostomum (Platyhelminthes, Macrostomida) from southern China. Zootaxa 4012, $120-134$.

Tan, G., Muffato, M., Ledergerber, C., Herrero, J., Goldman, N., Gil, M., Dessimoz, C., 2015. Current methods for automated filtering of multiple sequence alignments frequently worsen single-gene phylogenetic inference. Syst. Biol. 64 (5), 778-791.

Tekaia, F., 2016. Inferring orthologs: open questions and perspectives. Genomics Insights 9, GEI.S37925. https://doi.org/10.4137/GEI.S37925.

Templeton, A.R., Crandall, K.A., Sing, C.F., 1992. A cladistic analysis of phenotypic associations with haplotypes inferred from restriction endonuclease mapping and DNA sequence data. III. Cladogram estimation. Genetics 132, 619-633.

Tyler, S., Schilling, S., Hooge, M., Bush, L.F., 2006. Turbellarian taxonomic database.

Vanhove, M., Tessens, B., Schoelinck, C., Jondelius, U., Littlewood, T., Artois, T., Huyse, T., 2013. Problematic barcoding in flatworms: a case-study on monogeneans and rhabdocoels (Platyhelminthes). ZooKeys 365, 355-379.

Vellnow, N., Vizoso, D.B., Viktorin, G., Schärer, L., 2017. No evidence for strong cytonuclear conflict over sex allocation in a simultaneously hermaphroditic flatworm. BMC Evol. Biol. 17, 103.

Wang, A.-T., 2005. Three new species of the genus Macrostomum from China (Platyhelminthes, Macrostomida, Macrostomidae). Acta Zootaxonomica Sin. 30, 714-720.

Wang, L., Xin, F., Fang, C.-Y., Zhang, Y., Wang, A.-T., 2017. Two new brackish-water species of Macrostomum (Platyhelminthes, Macrostomida) from mangrove wetland in southern China. Zootaxa 4276, 107.

Wasik, K., Gurtowski, J., Zhou, X., Ramos, O.M., Delás, M.J., Battistoni, G., El Demerdash, O., Falciatori, I., Vizoso, D.B., Smith, A.D., Ladurner, P., Schärer, L., McCombie, W.R., Hannon, G.J., Schatz, M., 2015. Genome and transcriptome of the regeneration-competent flatworm, Macrostomum lignano. Proc. Natl. Acad. Sci. 112, $12462-12467$.

Waterhouse, R.M., Seppey, M., Simão, F.A., Manni, M., Ioannidis, P., Klioutchnikov, G., Kriventseva, E.V., Zdobnov, E.M., 2017. BUSCO applications from quality assessments to gene prediction and phylogenomics. Mol. Biol. Evol.

Wu, M., Chatterji, S., Eisen, J.A., Salemi, M., 2012. Accounting for alignment uncertainty in phylogenomics. PLoS ONE 7 (1), e30288. https://doi.org/10.1371/journal. pone.0030288. 
Wudarski, J., Egger, B., Ramm, S.A., Schärer, L., Ladurner, P., Zadesenets, K.S., Rubtsov, N.B., Mouton, S., Berezikov, E., 2020. The free-living flatworm Macrostomum lignano. EvoDevo 11 (1). https://doi.org/10.1186/s13227-020-001501.

Wudarski, J., Simanov, D., Ustyantsev, K., de Mulder, K., Grelling, M., Grudniewska, M., Beltman, F., Glazenburg, L., Demircan, T., Wunderer, J., Qi, W., Vizoso, D.B., Weissert, P.M., Olivieri, D., Mouton, S., Guryev, V., Aboobaker, A., Schärer, L., Ladurner, P., Berezikov, E., 2017. Efficient transgenesis and annotated genome sequence of the regenerative flatworm model Macrostomum lignano. Nat. Commun. 8,2120 .

Wunderer, J., Lengerer, B., Pjeta, R., Bertemes, P., Kremser, L., Lindner, H., Ederth, T., Hess, M.W., Stock, D., Salvenmoser, W., Ladurner, P., 2019. A mechanism for temporary bioadhesion. Proc. Natl. Acad. Sci. 116 (10), 4297-4306.

Xin, F., Zhang, S.-Y., Shi, Y.-S., Wang, L., Zhang, Y., Wang, A.-T., 2019. Macrostomum shenda and $M$. spiriger, two new brackish-water species of Macrostomum (Platyhelminthes: Macrostomorpha) from China. Zootaxa 4603:105.

Yang, Y., Smith, S.A., 2014. Orthology inference in nonmodel organisms using transcriptomes and low-coverage genomes: improving accuracy and matrix occupancy for phylogenomics. Mol. Biol. Evol. 31, 3081-3092.
Zadesenets, K.S., Jetybayev, I.Y., Schärer, L., Rubtsov, NB., 2020. Genome and karyotype reorganization after whole genome duplication in free-living flatworms of the genus Macrostomum. Int. J. Mol. Sci. 21 (2), 680. https://doi.org/10.3390/ijms21020680.

Zadesenets, K.S., Schärer, L., Rubtsov, N.B., 2017. New insights into the karyotype evolution of the free-living flatworm Macrostomum lignano (Platyhelminthes, Turbellaria). Sci. Rep. 7, 6066.

Zadesenets, K.S., Vizoso, D.B., Schlatter, A., Konopatskaia, I.D., Berezikov, E., Schärer, L., Rubtsov, N.B., 2016. Evidence for karyotype polymorphism in the free-living flatworm, Macrostomum lignano, a model organism for evolutionary and developmental biology. PLoS ONE 11, e0164915.

Zaharia, M., Bolosky, W.J., Curtis, K., Fox, A., Patterson, D., Shenker, S., Stoica, I., Karp, R.M., Sittler, T., 2011. Faster and more accurate sequence alignment with SNAP. ArXiv Prepr. ArXiv11115572.

Zhang, C., Rabiee, M., Sayyari, E., Mirarab, S., 2018. ASTRAL-III: polynomial time species tree reconstruction from partially resolved gene trees. BMC Bioinf. 19 (S6) https://doi.org/10.1186/s12859-018-2129-y.

Zhang, S., Shi, Y., Zeng, Z., Xin, F., Deng, L., 2021. Two new brackish-water species of Macrostomum (Platyhelminthes: Macrostomorpha) from China and their phylogenetic positions. Zool. Sci. 38. 